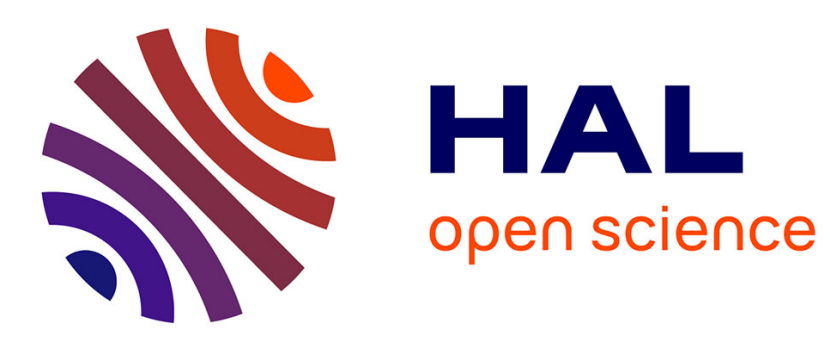

\title{
Convergence of utility indifference prices to the superreplication price in a multiple-priors framework
}

Romain Blanchard, Laurence Carassus

\section{To cite this version:}

Romain Blanchard, Laurence Carassus. Convergence of utility indifference prices to the superreplication price in a multiple-priors framework. 2018. hal-01883423

\section{HAL Id: hal-01883423 \\ https://hal.science/hal-01883423}

Preprint submitted on 28 Sep 2018

HAL is a multi-disciplinary open access archive for the deposit and dissemination of scientific research documents, whether they are published or not. The documents may come from teaching and research institutions in France or abroad, or from public or private research centers.
L'archive ouverte pluridisciplinaire HAL, est destinée au dépôt et à la diffusion de documents scientifiques de niveau recherche, publiés ou non, émanant des établissements d'enseignement et de recherche français ou étrangers, des laboratoires publics ou privés. 


\title{
Convergence of utility indifference prices to the superreplication price in a multiple-priors framework
}

\author{
Romain Blanchard, E.mail : romain.blanchard@etudiant.univ-reims.fr \\ LMR, Université Reims Champagne-Ardenne. \\ Laurence Carassus, E.mail : laurence.carassus@devinci.fr \\ Research Center, Léonard de Vinci Pôle universitaire \\ and LMR, Université Reims Champagne-Ardenne.
}

\begin{abstract}
This paper formulates an utility indifference pricing model for investors trading in a discrete time financial market under non-dominated model uncertainty. The investors preferences are described by strictly increasing concave random functions defined on the positive axis. We prove that under suitable conditions the multiple-priors utility indifference prices of a contingent claim converge to its multiple-priors superreplication price. We also revisit the notion of certainty equivalent for random utility functions and establish its relation with the absolute risk aversion.
\end{abstract}

Key words: Utility indifference price; Superreplication price; absolute risk aversion, Knightian uncertainty; multiple-priors, non-dominated model

AMS 2000 subject classification: Primary 91B70, 91B16, 91G20 ; secondary 91G10, 91B30, 28B20

JEL classification: C61, D81, G11, G13

\section{Introduction}

In this paper, we are examine different definitions of prices for a contingent claim and their relation in the context of uncertainty. Risk and uncertainty are at the heart of economic life, and modeling the way an agent will react to them is a central thematic of the economic research (see for instance (?)). By uncertainty we refer to Knightian uncertainty and we distinguish between the known unknown (risk) and unknown unknown (uncertainty) as introduced by F. Knight (?). In other words the agent cannot be certain about the choice of a given prior modelling the outcome of a situation. Issues related to uncertainty arise in various concrete situations in social science and economics, such as policy-making but also in many aspects of modern finance such as model risk when pricing and risk-managing complex derivatives products or capital requirement quantification when looking at regulation for banks and others financial entities. As illustrated for instance in the Ellsberg Paradox (see (?)), when facing uncertainty an agent displays uncertainty aversion: she tends to prefer a situation where the unknown unknown is reduced. This is the pendant of the risk aversion when the agent faces only risk. And it is well known that if one wants to represent the preferences of the agent in this context, the axiomatic of the von Neumann and Morgenstern expected utility criterium (see (?)) are not verified even if the Savage's 
extension (see (?)), where subjective probability measures depending on each agent are introduced, is considered. In this paper, we follow the pioneering approach introduced by (?) where under suitable axiomatic on the investor preferences, the utility functional is of the form of a worst case expected utility: $\inf _{P \in \mathcal{Q}} E_{P} U(\cdot, X)$, where $\mathcal{Q}$ is the set of all possible probability measures representing the agent's beliefs. Note that this approach can also be used for robustness considerations where a set of models resulting from small perturbations of the initial reference model is taken. This is related for instance to the work of (?) where a term corresponding to the relative entropy given a certain reference probability measure is added to the utility functional. The framework of (?) was extended by (?) who introduced a penalty term to the utility functional. Finally, (?) represent the preferences by a more general functional $\inf _{P \in \mathcal{Q}} G\left(E_{P} U(X), P\right)$ where $G$ is a so-called uncertainty index reflecting the decision-maker's attitudes toward uncertainty.

From an economic and practical point of view an important and welcome feature is to consider a set of probability measures $\mathcal{Q}$ which is non-dominated. This means that no probability measure determines the set of events that can happen or not. The relevance of this idea is illustrated by the concrete example of volatility uncertainty, see (?), (?)) and (?). However this increases significantly the mathematical difficulty as some of the classical tools of probability theory such as conditional expectation or essential supremum are ill-suited (since they are defined with respect to a given probability measure). These type of issues have contributed to the development of innovative mathematical tools such as quasi-sure stochastic analysis, non-linear expectations, G-Brownian motions. On these topics, we refer amongst others to (?), (?) or (?).

The No Arbitrage (NA) notion is central to many problems in quantitative finance. It asserts that starting from a zero wealth it is not possible to reach a positive one (non negative almost surely and strictly positive with strictly positive probability). The characterisation of this condition or of the No Free Lunch condition is called the Fundamental Theorem of Asset Pricing (FTAP in short) and makes the link between those notions and the existence of equivalent risk-neutral probability measures (also called martingale measures or pricing measures) which are equivalent probability measures that turn the (discounted) asset price process into a martingale. This was initially formalised in (?), (?) and (?) while (?) obtain the FTAP in a general discretetime setting under the NA condition. The literature on the subject is huge and we refer to (?) for a general overview. The martingale measures allow for pricing issues and another fundamental result, the Superhedging Theorem (see for instance (?) or (?)) relates those pricing measures to the set of no-arbitrage prices for a given contingent claim. The so-called superreplication price is the minimal amount needed for an agent selling a claim in order to superreplicate it by trading in the market. To the best of our knowledge it was first introduced in (?) in the context of transaction costs. This is a hedging price with no risk but unfortunately it is not always of practical use as it is often too onerous: for example the superreplication price of a call option may be equal to the underlying initial price in an incomplete market (see (?)).

All these concepts have seen a renewed interest in the context of uncertainty, see amongst others (?), (?), (?), (?), (?), (?), (?), (?) and (?). In this paper, we have chosen to work under the discrete-time framework introduced in (?). We outline briefly in Sections ?? and ?? some of the interesting features of this framework, in particular with respect to time-consistency.

Utility indifference price or reservation price was first introduced in ? in the 
context of transaction costs as the minimal amount of money to be paid to an agent selling a contingent claim $G$ so that added to her initial capital, her utility when selling $G$ and hedging it by trading dynamically in the market is greater or equal to the one she would get without selling this product (see Definition ??). This notion of price is linked to certainty equivalent and takes into account the preferences of the agent. Unfortunately it is very difficult to compute outside the constant absolute risk aversion case (i.e. for exponential utility functions).

Note also that the notions of superreplication or utility indifference pricing can be related to the concept of risk measures introduced by (?) as illustrated in Propositions ?? and ??. For more details on risk measures in the context of multiple-priors we refer for instance to (?), (?) and more recently (?).

Intuitively speaking, the utility indifference price allows for some preference base risk-seeking behavior while the superreplication price corresponds to a totally risk averse agent. In the paper we investigate the effect of increasing risk aversion on utility based prices: when absolute risk aversion tends to infinity, the reservation price should tend to the superreplication price. Our main contribution is presented in Theorem ??. We consider a sequence of investors whose preferences are represented by a sequence of random utility functions (see Definition ??) trading in a discrete-time financial market in the presence of uncertainty. We establish that under suitable conditions, the multiple-priors utility indifference prices of a given contingent claim (for the seller) converge to its multiple-priors superreplication price. For non random utility functions (see Theorem ??) those conditions are implied by the convergence to infinity of the absolute risk-aversion (see Definition ??) of the agents.

In the mono-prior case for constant absolute risk averse agents, the convergence of reservation prices to the superreplication price was shown by ? for Brownian models and in ? in a general semimartingale setting. A nonexponential case was treated in?, but with severe restrictions on the utility functions. The case of general utilities was considered in ? in discrete-time market models and in ? for continuous time ones.

To the best of our knowledge Theorem ?? is the first general asymptotic result in the multiple-priors framework. Note that simultaneously ? obtains some convergence result for a different utility based price and constant absolute risk averse agents. In Theorem ??, we apply Theorem ?? and prove that the convergence result occurs for a large class of non-random utility functions while in Proposition ?? we obtain the pendant asymptotic result for the multiple-priors subreplication and utility indifference buyer prices. We also show the convergence of the associated risk measures (see Proposition ??).

To solve our problem, we use some arguments of (?) that are adapted to our multiple-priors framework together with results of (?, Theorems 2.2 and 2.3). We also use some elements of quasi-sure stochastic analysis as developed in (?) and (?).

We revisit as well, in a static context, the notion of certainty equivalent introduced in (?). We extend it for random utility functions and in the presence of multiple-priors and we establish that the absolute risk aversion allows the ranking of the multiplepriors certainty equivalent despite the presence of uncertainty aversion (see Proposition ??). This part is also related to (?) where an alternative notion of indifference buyer (and seller) prices are introduced for non-random utility functions in a static setting under the representation of (?).

The article is structured as follows: Section ?? presents our framework, some definitions and results needed in the rest of the paper. Section ?? revisits the link between certainty equivalent and absolute risk aversion in our set-up. Section ?? 
presents the main theorem on the convergence of the utility indifference prices to the superreplication price. Finally, Section ?? contains the remaining proofs and technical results.

\section{The model}

This section presents our multiple-priors framework and the definitions of the suband superreplication prices and of the utility buyer and seller indifference prices.

\subsection{Uncertainty modelisation}

We model uncertainty as in (?) and (?) and use similar notations as in (?).

For any Polish space $X$ (i.e complete and separable metric space), we denote by $\mathcal{B}(X)$ its Borel sigma-algebra and by $\mathfrak{P}(X)$ the set of all probability measures on $(X, \mathcal{B}(X))$. For some $P \in \mathfrak{P}(X)$ fixed, we denote by $\mathcal{B}_{P}(X)$ the completion of $\mathcal{B}(X)$ with respect to $P$ and we introduce the universal sigma-algebra defined by $\mathcal{B}_{c}(X):=\bigcap_{P \in \mathfrak{P}(X)} \mathcal{B}_{P}(X)$. It is clear that $\mathcal{B}(X) \subset \mathcal{B}_{c}(X)$. In the rest of the paper, we will use the same notation for $P \in \mathfrak{P}(X)$ and for its (unique) extension on $\mathcal{B}_{c}(X)$. For a given $\mathcal{Q} \subset \mathfrak{P}(X)$, a set $N \subset X$ is called a $\mathcal{Q}$-polar if for all $P \in \mathcal{Q}$, there exists some $A_{P} \in \mathcal{B}_{c}(X)$ such that $P\left(A_{P}\right)=0$ and $N \subset A_{P}$. We say that a property holds true $\mathcal{Q}$-quasi-surely (q.s.), if it is true outside a $\mathcal{Q}$-polar set. Finally we say that a set is of $\mathcal{Q}$-full measure if its complement is a $\mathcal{Q}$-polar set. A function $f: X \rightarrow Y$ (where $Y$ is an other Polish space) is universally-measurable or $\mathcal{B}_{c}(X)$-measurable (resp. Borel-measurable or $\mathcal{B}(X)$ measurable) if for all $B \in \mathcal{B}(Y), f^{-1}(B) \in \mathcal{B}_{c}(X)$ (resp. $f^{-1}(B) \in \mathcal{B}(X)$ ). Similarly we will speak of universally-adapted or universally-predictable (resp. Borel-adapted or Borel-predictable) processes.

We fix a time horizon $T \in \mathbb{N}$ and introduce a sequence $\left(\Omega_{t}\right)_{1 \leq t \leq T}$ of Polish spaces. We denote by $\Omega^{t}:=\Omega_{1} \times \cdots \times \Omega_{t}$, with the convention that $\Omega^{0}$ is reduced to a singleton. An element of $\Omega^{t}$ will be denoted by $\omega^{t}=\left(\omega_{1}, \ldots, \omega_{t}\right)=\left(\omega^{t-1}, \omega_{t}\right)$ for $\left(\omega_{1}, \ldots, \omega_{t}\right) \in$ $\Omega_{1} \times \cdots \times \Omega_{t}$ and $\left(\omega^{t-1}, \omega_{t}\right) \in \Omega^{t-1} \times \Omega_{t}$ (to avoid heavy notation we drop the dependency in $\left.\omega_{0}\right)$. For all $0 \leq t \leq T-1$, we denote by $\mathcal{S} K_{t+1}$ the set of universally-measurable stochastic kernel on $\Omega_{t+1}$ given $\Omega^{t}$, see (?, Definition $7.12 \mathrm{p} 134$, Lemma $7.28 \mathrm{p} 174$ ). Fix some $1 \leq t \leq T, P_{t-1} \in \mathfrak{P}\left(\Omega^{t-1}\right)$ and $p_{t} \in \mathcal{S} K_{t}$. Using Fubini's Theorem, see (?, Proposition $7.45 \mathrm{p} 175)$, we define a probability measure on $\mathcal{B}_{c}\left(\Omega^{t}\right)$ as follows

$$
P_{t-1} \otimes p_{t}(A):=\int_{\Omega^{t-1}} \int_{\Omega_{t}} 1_{A}\left(\omega^{t-1}, \omega_{t}\right) p_{t}\left(d \omega_{t}, \omega^{t-1}\right) P_{t-1}\left(d \omega^{t-1}\right),
$$

where $A \in \mathcal{B}_{c}\left(\Omega^{t}\right)$. To model the uncertainty we consider a family of random sets $\mathcal{Q}_{t+1}: \Omega^{t} \rightarrow \mathfrak{P}\left(\Omega_{t+1}\right)$, for all $0 \leq t \leq T-1$. The set $\mathcal{Q}_{t+1}\left(\omega^{t}\right)$ can be seen as the set of all possible models for the $t+1$-th period given the state $\omega^{t}$ until time $t$. From

the random sets $\left(\mathcal{Q}_{t+1}\right)_{0 \leq t \leq T-1}$ we build the sets of probability measures $\left(\mathcal{Q}^{t}\right)_{1 \leq t \leq T}$ where $\mathcal{Q}^{t}$ governs the market until time $t$ and determines which events are relevant or not in $\mathcal{B}_{c}\left(\Omega^{t}\right)$. To do that, as in (?), (?), we have to make the following assumption which is now classical in the recent litterature on multiple-priors models.

Assumption 2.1 For all $0 \leq t \leq T-1, \mathcal{Q}_{t+1}$ is a non-empty and convex valued random set such that

$$
\operatorname{Graph}\left(\mathcal{Q}_{t+1}\right)=\left\{\left(\omega^{t}, P\right) \in \Omega^{t} \times \mathfrak{P}\left(\Omega_{t+1}\right), P \in \mathcal{Q}_{t+1}\left(\omega^{t}\right)\right\}
$$

is an analytic set. 
Recall that an analytic set is the continuous image of some Polish space, see (?, Theorem 12.24 p447), see also (?, Chapter 7) for more details on analytic sets. Assumption ?? allows to apply the Jankov-von Neumann Theorem (see for example (?, Proposition $7.49 \mathrm{p} 182)$ ) and to get some universally-measurable selector $q_{t+1}$ of $\mathcal{Q}_{t+1}$. Then, for each time $1 \leq t \leq T$, the set $\mathcal{Q}^{t} \subset \mathfrak{P}\left(\Omega^{t}\right)$ is completely determined by the random sets of one-step models $\mathcal{Q}_{s+1}$ (for $s=0, \cdots, t-1$ ) in the following way

$\mathcal{Q}^{t}:=\left\{Q_{1} \otimes q_{2} \otimes \cdots \otimes q_{t}, Q_{1} \in \mathcal{Q}_{1}, q_{s+1} \in \mathcal{S} K_{s+1}, q_{s+1}\left(\cdot, \omega^{s}\right) \in \mathcal{Q}_{s+1}\left(\omega^{s}\right) Q_{s}\right.$-a.s. $\left.s \in\{1, \ldots, t-1\}\right\}$,

where we denote $Q_{s}:=Q_{1} \otimes q_{2} \otimes \cdots \otimes q_{s}$ for any $2 \leq s \leq t$.

The technical Assumption ?? plays a key role to obtain measurability properties required to prove the FTAP, the Superreplication Theorem and also to apply a dynamic programming procedure in multiple-priors utility maximisation problem (see for instance (?), (?), (?) or (?)). More precisely, if $X_{t+1}: \Omega^{t+1} \rightarrow \mathbb{R}$ is lower-semianalytic (see (?, Definition 7.21)), then $X_{t}: \Omega^{t} \rightarrow \mathbb{R}$ defined by

$$
X_{t}\left(\omega^{t}\right)=\inf _{P \in \mathcal{Q}_{t+1}\left(\omega^{t}\right)} \int_{\Omega_{t+1}} X_{t+1}\left(\omega^{t}, \omega_{t+1}\right) P\left(d \omega_{t+1}\right)
$$

remains lower-semianalytic. More generally, this framework allows to construct families of dynamic sublinear expectations (see (?, Lemma 4.10) and also (?)). For similar issues in the continuous-time setting we also refer also amongst others to (?), (?) and (?). Apart from Assumption ??, we make no specific assumptions on the set of priors: $\mathcal{Q}^{T}$ is neither assumed to be dominated by a given reference probability measure nor to be weakly compact. For example, in the continuous-time case, dominated set of priors can arise when there is uncertainty on the drift of the underlying process while non-dominated set of priors may arise if there is uncertainty on the volatility of this process (see for example (?)). In the case of volatility uncertainty, the corresponding set is however weakly compact (see for instance (?), (?), (?, Proposition 3) and also (?)).

We focus briefly on the time-consistency issue: how are the agent decisions or risk evaluations at different times interrelated once the information has been updated. Roughly speaking, time-consistency means that a decision taken tomorrow will satisfies today's objective. Recall that this issue appears already in mono-prior setting, in the study of dynamic risk measures for instance, and is linked with the law of iterated conditional expectations and the dynamic programming principle. We refer to the surveys (?) and (?) for detailed overviews.

Now when introducing multiple-priors one has to be even more careful with timeconsistency. In (?, Appendix D) a simple example illustrates what can happen if one is not cautious on the structure of the initial set of priors: one cannot hope to find an optimal solution using the dynamic programming principle when trying for instance to maximise a worst case expected utility problem. To deal with this, one has to assume that the set of prior is stable under pasting which roughly means that different priors can be mixed together (see (?, Assumption 4)). Given (??), it is clear that our set of priors are stable under pasting. Indeed, if $Q^{1}, Q^{2} \in \mathcal{Q}^{T}$ with $Q^{1}=Q_{1}^{1} \otimes q_{2}^{1} \otimes \cdots \otimes q_{T}^{1}$, $Q^{2}=Q_{1}^{2} \otimes q_{2}^{2} \otimes \cdots \otimes q_{T}^{2}$, then $R:=Q_{1}^{1} \otimes q_{2}^{1} \cdots \otimes q_{t-1}^{1} \otimes q_{t}^{2} \otimes \cdots \otimes q_{T}^{2} \in \mathcal{Q}^{T}$ for all $2 \leq t \leq T-1$. In a sense, the set $\mathcal{Q}^{T}$ is large enough (unlike in the example considered in (?, Appendix D) ). In (?, Definition 3.1) the equivalent notion of rectangularity is introduced (see also (?, Sections 3, 4) for more details and a graphical interpretation). 
For $1 \leq t \leq T$ fixed, we introduce the following spaces

$$
\begin{aligned}
\mathcal{W}_{t}^{0} & :=\left\{X: \Omega^{t} \rightarrow \mathbb{R} \cup\{ \pm \infty\}, \mathcal{B}_{c}\left(\Omega^{t}\right) \text {-measurable }\right\}, \\
\mathcal{W}_{t}^{\infty} & :=\mathcal{W}_{t}^{0} \cap\left\{X, \exists M \geq 0,|X| \leq M \mathcal{Q}^{t} \text {-q.s. }\right\} .
\end{aligned}
$$

Finally, we will add a superscript ${ }^{+}$when considering non-negative elements (it will be also used for denoting positive parts).

\subsection{The traded assets and the trading strategies}

Let $S:=\left\{S_{t}, 0 \leq t \leq T\right\}$ be a universally-adapted $d$-dimensional process where for $0 \leq t \leq T, S_{t}=\left(S_{t}^{i}\right)_{1 \leq i \leq d}$ represents the price of $d$ risky securities in the financial market in consideration. To solve measurability issues, we make the following assumption already present in (?) and (?).

Assumption 2.2 The price process $S$ is Borel-adapted.

Trading strategies are represented by universally-adapted $d$-dimensional processes $\phi:=\left\{\phi_{t}, 1 \leq t \leq T\right\}$ where for all $1 \leq t \leq T, \phi_{t}=\left(\phi_{t}^{i}\right)_{1 \leq i \leq d}$ represents the investor's holdings in each of the $d$ assets at time $t$. The set of trading strategies is denoted by $\Phi$.

We assume that trading is self-financing and that the riskless asset's price is constant equal to 1 . The value at time $t$ of a portfolio $\phi$ starting from initial capital $x \in \mathbb{R}$ is given by $V_{t}^{x, \phi}=x+\sum_{s=1}^{t} \phi_{s} \Delta S_{s}$.

\subsection{Multiple-priors no-arbitrage condition}

As already eluded to in the introduction, the issue of no-arbitrage in the context of uncertainty has seen a renewed interest. In this paper we follow the definition introduced by (?) that we recall below. We outline briefly some of the interesting feature of this definition. First it looks like a natural and intuitive extension of the classical mono-prior arbitrage condition. This argument is strengthened by the FTAP generalisation proved by (?). Under appropriate measurability conditions the $N A\left(\mathcal{Q}^{T}\right)$ is equivalent to the following: for all $Q \in \mathcal{Q}^{T}$, there exists some $P \in \mathcal{R}^{T}$ such that $Q \ll P$ where

$$
\mathcal{R}^{T}:=\left\{P \in \mathfrak{P}\left(\Omega^{T}\right), \exists Q^{\prime} \in \mathcal{Q}^{T}, P \ll Q^{\prime} \text { and } P \text { is a martingale measure }\right\} .
$$

The classical notion of equivalent martingale measure is replaced by the fact that for all priors $Q \in \mathcal{Q}^{T}$, there exists a martingale measure $P$ such that $Q$ is absolutely continuous with respect to $P$ and one can find an other prior $Q^{\prime} \in \mathcal{Q}^{T}$ such that $P$ is absolutely continuous with respect to $Q^{\prime}$. The extension in the same multiple-priors setting of the Superhedging Theorem and subsequent results on worst-case expected utility maximisation (see (?), (?), (?) and (?) is an other convincing element.

Assumption 2.3 The $N A\left(\mathcal{Q}^{T}\right)$ condition holds true if for $\phi \in \Phi, V_{T}^{0, \phi} \geq 0 \mathcal{Q}^{T}$-q.s. implies that $V_{T}^{0, \phi}=0 \mathcal{Q}^{T}$-q.s.

For the convenience of the reader we recall the following definition and proposition from (?) concerning the multiple-priors conditional support of the price increments or more precisely of its affine hull (denoted by Aff from now). 
Definition 2.4 For all $0 \leq t \leq T-1$ we define the random set $D^{t+1}: \Omega^{t} \rightarrow \mathbb{R}^{d}$ by

$$
D^{t+1}\left(\omega^{t}\right):=\operatorname{Aff}\left(\bigcap\left\{A \subset \mathbb{R}^{d}, \text { closed, } P_{t+1}\left(\Delta S_{t+1}\left(\omega^{t}, .\right) \in A\right)=1, \forall P_{t+1} \in \mathcal{Q}_{t+1}\left(\omega^{t}\right)\right\}\right) .
$$

Proposition 2.5 Assume that the $N A\left(\mathcal{Q}^{T}\right)$ condition and Assumptions ??, ?? hold true. Then for all $0 \leq t \leq T-1$, there exists some $\mathcal{Q}^{t}$-full measure set $\Omega_{N A}^{t} \in \mathcal{B}_{c}\left(\Omega^{t}\right)$ such that for all $\omega^{t} \in \Omega_{N A}^{t}, D^{t+1}\left(\omega^{t}\right)$ is a vector space. For all $\omega^{t} \in \Omega_{N A}^{t}$ there exists $\alpha_{t}\left(\omega^{t}\right)>0$ such that for all $h \in D^{t+1}\left(\omega^{t}\right)$ there exists $P_{h} \in \mathcal{Q}_{t+1}\left(\omega^{t}\right)$ satisfying

$$
P_{h}\left(\frac{h}{|h|} \Delta S_{t+1}\left(\omega^{t}, .\right)<-\alpha_{t}\left(\omega^{t}\right)\right)>\alpha_{t}\left(\omega^{t}\right) .
$$

Note that in (?), the equivalence between Assumption ?? and condition ?? is established. In the case where there is only one risky asset and one period, the interpretation of (??) is straightforward. It simply means that there exists a prior (i.e. some probability $P^{+}$) for which the price of the risky asset increases enough and an other one $\left(P^{-}\right)$for which the price decreases, i.e $P^{ \pm}(\mp \Delta S(\cdot)<-\alpha)>\alpha$ where $\alpha>0$. The number $\alpha$ serves as a measure of the gain/loss and of their size. Note that for an agent buying or selling some quantity of the risky asset, there is always a prior in which she is exposed to a potential loss

For all $x \geq 0$, we introduce the set of terminal wealth including the possibility of throwing away money defined by

$$
\mathcal{C}_{x}^{T}:=\left\{V_{T}^{x, \phi}, \phi \in \Phi\right\}-\mathcal{W}_{T}^{0,+} .
$$

In the sequel we will write $X \in \mathcal{C}_{x}^{T}$ if there exists some $\phi \in \Phi$ and $Z \in \mathcal{W}_{T}^{0,+}$ such that $X=V_{T}^{x, \phi}-Z \mathcal{Q}^{T}$ q.s. Under the assumptions of Lemma ?? (which will be crucial in Section ??), the set $\mathcal{C}_{x}^{T}$ has a classical closure property (in the $\mathcal{Q}^{T}$ quasi-sure sense, see (?, Theorem 2.2)).

Lemma 2.6 Assume that Assumptions ?? and ?? hold true. Fix some $z \geq 0$ and let $B \in \mathcal{W}_{T}^{0}$ such that $B \notin \mathcal{C}_{z}^{T}$. Then there exists some $\varepsilon>0$ such that

$$
\inf _{\phi \in \Phi} \sup _{P \in \mathcal{Q}^{T}} P\left(V_{T}^{z, \phi}<B-\varepsilon\right)>\varepsilon .
$$

Proof. Assume that (??) does not hold true. Then, for all $n \geq 1$, there exist some $\phi_{n} \in \Phi$ such that $P\left(V_{n}<B-\frac{1}{n}\right) \leq \frac{1}{n}$ for all $P \in \mathcal{Q}^{T}$, where $V_{n}:=V_{T}^{z, \phi_{n}}$. Set $K_{n}:=$ $\left(V_{n}-\left(B-\frac{1}{n}\right)\right) 1_{\left\{V_{n} \geq B-\frac{1}{n}\right\}} \in \mathcal{W}_{T}^{0,+}$, then $V_{n}-K_{n} \in \mathcal{C}_{z}^{T}$. Moreover $P\left(\left|V_{n}-K_{n}-B\right|>\right.$ $\left.\frac{1}{n}\right)=P\left(V_{n}<B-\frac{1}{n}\right) \leq \frac{1}{n}$ for all $P \in \mathcal{Q}^{T}$. Thus $\lim _{n \rightarrow \infty} \sup _{P \in \mathcal{Q}^{T}} P\left(\left|V_{n}-K_{n}-B\right|>\frac{1}{n}\right)=0$ and using Proposition ??, there exists a subsequence $\left(n_{k}\right)_{k \geq 1}$ such that $\left(V_{n_{k}}-K_{n_{k}}\right)_{k \geq 1}$ converges to $B \mathcal{Q}^{T}$-q.s. (i.e. on a $\mathcal{Q}^{T}$-full measure set). Applying (?, Theorem 2.2), we get that $B \in \mathcal{C}_{z}^{T}$, a contradiction.

Remark 2.7 Note that to apply (?, Theorem 2.2) we do not need Assumptions ?? to hold true. Similarly it applies under a weaker assumption than Assumption ??.

\subsection{Multiple-priors superreplication and subreplication prices}

The multiple-priors superreplication price is the minimal initial amount that an agent will ask for delivering some contingent claim $G \in \mathcal{W}_{T}^{0}$ so that she is fully hedged at $T$ when trading in the market. The multiple-priors subreplication price 
is the maximal amount an agent will accept to pay in order to receive some contingent claim while being fully hedge at $T$ by trading in the market. Note that the superreplication price is a seller price while the subreplication price is a buyer price. We also introduce the set of strategies which dominate $G \mathcal{Q}^{T}$-q.s. starting from a given wealth $x \in \mathbb{R}$

$$
\mathcal{A}(G, x):=\left\{\phi \in \Phi, V_{T}^{x, \phi} \geq G \mathcal{Q}^{T} \text { q.s. }\right\}
$$

Definition 2.8 Let $G \in \mathcal{W}_{T}^{0}$. The multiple-priors superreplication price of $G$ is defined by

$$
\pi(G):=\inf \{z \in \mathbb{R}, \mathcal{A}(G, z) \neq \emptyset\},
$$

and $\pi(G)=+\infty$ if $\mathcal{A}(G, z)=\emptyset$ for all $z \in \mathbb{R}$. The multiple-priors subreplication price of $G$ is defined by

$$
\pi^{s u b}(G):=\sup \{z \in \mathbb{R}, \mathcal{A}(-G,-z) \neq \emptyset\},
$$

and $\pi^{s u b}(G)=-\infty$ if $\mathcal{A}(-G,-z)=\emptyset$ for all $z \in \mathbb{R}$.

We recall now for the convenience of the reader (?, Theorem 2.3) slightly adapted to our setup (the same comment as in Remark ?? applies)

Theorem 2.9 Assume that Assumptions ?? and ?? hold true and let $G \in \mathcal{W}_{T}^{0}$ be fixed. Then $\pi(G)>-\infty$ and $\mathcal{A}(G, \pi(G)) \neq \emptyset$.

If $G$ is replicable, i.e if there exists some $x_{G}$ and $\phi_{G} \in \Phi$ such that $G=V_{T}^{x_{G}, \phi_{G}}$ $\mathcal{Q}^{T}$-q.s., then $\pi(G)=x_{G}=\pi\left(V_{T}^{x_{G}, \phi_{G}}\right)$. Note that under some measurability assumption on $G$, the Superreplication Theorem is still true: $\pi(G)=\sup _{P \in \mathcal{R}^{T}} E_{P} G$, see (?, Superhedging Theorem) and (??) for the definition of $\mathcal{R}^{T}$.

Note that if $G \in \mathcal{W}_{T}^{\infty}$, it is clear that $\pi(G) \leq\|G\|_{\infty}$. This is the case if $G$ represents the payoff of a put option or a digital option but not for a call option. This illustrates that the case $G \in \mathcal{W}_{T}^{\infty}$ can be sometimes too restrictive especially in a multiple-priors setting and explains why in the rest of the paper, we will try to avoid results limited to $\mathcal{W}_{T}^{\infty}$. The price to pay is often related to integrability issues. The next lemma resumes some basic results on superreplication prices.

Lemma 2.10 Let $G \in \mathcal{W}_{T}^{0}$ then $\pi^{\text {sub }}(G)=-\pi(-G)$. Moreover $\pi(G)=+\infty$ if and only if $\mathcal{A}(G, z)=\emptyset$ for all $z \in \mathbb{R}$. If Assumption ?? holds true then $\pi(0)=0$. If furthermore Assumption ?? holds true and $G \in \mathcal{W}_{T}^{0,+}$, then $\pi(G)=0$ implies that $G=0 \mathcal{Q}^{T}$-q.s.

Proof. The two first assertions are clear. By definition $\pi(0) \leq 0$. Assume that $\pi(0)<0$ and let $\varepsilon>0$ such that $\pi(0)<-\varepsilon$. Then there exists some $\phi \in \Phi$ such that $V_{T}^{0, \phi} \geq \varepsilon>0$ $\mathcal{Q}^{T}$-q.s. a contradiction with $N A\left(\mathcal{Q}^{T}\right)$. For the last assertion, assume that there exists some $P \in \mathcal{Q}^{T}$ such that $P(G(\cdot)>0)>0$. Using Theorem ?? there exists $\phi_{0} \in \Phi$ such that $V_{T}^{\pi(G), \phi_{0}} \geq G \mathcal{Q}^{T}$-q.s. Thus $P\left(V_{T}^{0, \phi_{0}}>0\right)>0$ which contradicts $N A\left(\mathcal{Q}^{T}\right)$.

We now turn to some pricing rules which takes into account the preferences of the agents. 


\subsection{Utility functions and utility indifference prices}

In this paper we focus on utility function defined on the half-real line whose definition follows.

Definition 2.11 A random utility function $U: \Omega^{T} \times(0, \infty) \rightarrow \mathbb{R} \cup\{-\infty\}$ satisfies the following conditions

i) for every $x>0, U(\cdot, x): \Omega^{T} \rightarrow \mathbb{R}$ is universally-measurable,

ii) for all $\omega^{T} \in \Omega^{T}, U\left(\omega^{T}, \cdot\right):(0, \infty) \rightarrow \mathbb{R}$ is concave, strictly increasing and twice continuously differentiable on $(0, \infty)$.

We extend $U$ by (right) continuity in 0 and set $U(\cdot, x)=-\infty$ if $x<0$.

Example 2.12 We give some concrete examples of random utility functions. The first one arises if the agent analyzes her gain or loss with respect to a (random) reference point $B$ rather than with respect to zero has suggested for instance by ?. Formally, let $\bar{U}$ be a non-random function satisfying Definition ?? and $B \in \mathcal{W}_{T}^{\infty,+}$. Set for all $\omega^{T} \in \Omega^{T}, x \geq 0, U\left(\omega^{T}, x\right)=\bar{U}\left(x+\|B\|_{\infty}-B\left(\omega^{T}\right)\right)$ and $U\left(\omega^{T}, x\right)=-\infty$ for $x<0$. Then it is clear that $U$ satisfies also the condition of Definition ??.

The second example allows to consider random absolute risk aversion (see Definition ?? for the precise statement of this concept). The idea is to use classical utility functions but with random coefficients. For example, we can consider $U\left(\omega^{T}, x\right)=x^{\beta_{1}\left(\omega^{T}\right)}$ or $U\left(\omega^{T}, x\right)=-e^{-\beta_{2}\left(\omega^{T}\right) x}$ for $x \geq 0$ (and $U(\cdot, x)=-\infty$ for $x<0$ ) where $\beta_{1}, \beta_{2} \in \mathcal{W}_{T}^{0}$ and $0<\beta_{1}(\cdot)<1, \beta_{2}(\cdot)>0 \mathcal{Q}^{T}$-q.s. We can imagine various situations for $\beta_{2}$ (which can be easily adapted for $\beta_{1}$ ): $\beta_{2}$ can be uniformly distributed on $\left[\beta_{\min }^{P}, \beta_{\max }^{P}\right]$ for all $P \in \mathcal{Q}^{T}$ (with $\beta_{\max }^{P} \geq \beta_{\min }^{P}>0$ ) or alternatively it could follow a Poisson law of parameter $\lambda_{P}>0$ for all $P \in \mathcal{Q}^{T}$.

We now turn to pricing issues and first define some particular sets of strategies for a claim $G \in \mathcal{W}_{T}^{0}$ and some $x \in \mathbb{R}$ (recall (??))

$$
\begin{aligned}
\Phi(U, G, x) & :=\left\{\phi \in \Phi, E_{P} U^{+}\left(\cdot, V_{T}^{x, \phi}(\cdot)-G(\cdot)\right)<+\infty, \forall P \in \mathcal{Q}^{T}\right\} \\
\mathcal{A}(U, G, x) & :=\Phi(U, G, x) \cap \mathcal{A}(G, x) .
\end{aligned}
$$

Note that for $x \geq \pi(G), \mathcal{A}(U, G, x)$ might be empty. Indeed, from Theorem ?? there exists some $\phi \in \mathcal{A}(G, x)$, but $\phi$ might not belong to $\Phi(U, G, x)$. In Lemma ?? we will prove that under suitable conditions, $\mathcal{A}(G, x)=\mathcal{A}(U, G, x)$ for all $x \geq 0$. This is the reason why in $\Phi(U, G, x)$ we do not consider strategies such that $E_{P} U^{-}\left(\cdot, V_{T}^{x, \phi}(\cdot)-G(\cdot)\right)<\infty$.

We now introduce the quantity $u(G, x)$ which represents the maximum worst-case expected utility starting from initial capital $x$ and delivering $G$ at the terminal date

$$
u(G, x):=\sup _{\phi \in \mathcal{A}(U, G, x)} \inf _{P \in \mathcal{Q}^{T}} E_{P} U\left(\cdot, V_{T}^{x, \phi}(\cdot)-G(\cdot)\right),
$$

where $u(G, x)=-\infty$ if $\mathcal{A}(U, G, x)=\emptyset$.

We are now in position to define the (seller) multiple-priors utility indifference price or reservation price, which generalizes in the presence of uncertainty, the concept introduced by ?. It represents the minimal amount of money to be paid to an agent selling a contingent claim $G$ so that added to her initial capital, her multiple-priors utility when selling $G$ and hedging it by trading dynamically in the market is greater or 
equal than the one she would get without selling this product. Similarly the (buyer) multiple-priors utility indifference price represents the maximum amount of money an agent is ready to pay in order to buy $G$ so that subtracted to her initial capital, her multiple-priors expected utility when buying $G$ and hedging it by trading dynamically in the market is greater or equal than the one she would get without buying this product.

Definition 2.13 Let $G \in \mathcal{W}_{T}^{0}$ be a contingent claim. The (seller) multiple-priors utility indifference price is given by

$$
p(G, x):=\inf \{z \in \mathbb{R}, u(G, x+z) \geq u(0, x)\},
$$

where we set $p(G, x)=+\infty$ if $u(G, x+z)<u(0, x)$, for all $z \in \mathbb{R}$. The (buyer) multiplepriors utility indifference price is given by

$$
p^{B}(G, x):=\sup \{z \in \mathbb{R}, u(-G, x-z) \geq u(0, x)\},
$$

where we set $p^{B}(G, x)=-\infty$ if $u(-G, x-z)<u(0, x)$, for all $z \in \mathbb{R}$.

It is easy to see that $p^{B}(G, x)=-p(-G, x)$. We will see in Lemma ??, that under suitable integrability conditions $p(G, x) \leq \pi(G)$, for all $G \in \mathcal{W}_{T}^{0,+}$. Whatever the preference of the agent is, she will always evaluate a reservation price which is lower than the superreplication price. The superreplication price is, in the sense that we will define below, the price corresponding to an infinite absolute risk averse agent. The following proposition presents some other properties.

Proposition 2.14 We fix some $x \geq 0$ and assume that Assumptions ?? and ?? hold true and that $u(0, x)>-\infty$.

1. Let $G \in \mathcal{W}_{T}^{0}$. Then $p(G, x) \geq \pi(G)-x>-\infty, p^{B}(G, x) \leq \pi^{s u b}(G)+x<\infty$. In particular $-x \leq p(0, x) \leq 0$.

2. Let $G \in \mathcal{W}_{T}^{0,+}$ and assume that $E_{P} U^{+}\left(\cdot, V_{T}^{x, \phi}(\cdot)\right)<+\infty, \forall \phi \in \Phi, \forall P \in \mathcal{Q}^{T}$. Then $p(G, x) \geq p(0, x)$.

3. If $u(0, x-\delta)<u(0, x)$ for all $\delta>0$, then $p(0, x)=p^{B}(0, x)=0$.

Proof. 1. For any $G \in \mathcal{W}_{T}^{0}$ since Assumptions ?? and ?? hold true, Theorem ?? yields to $\pi(G)>-\infty$. Let $z \in \mathbb{R}$ be such that $x+z<\pi(G)$. By definition of $\pi(G), \mathcal{A}(G, x+z)=\emptyset$ and thus $u(G, x+z)=-\infty$ (see (??)). This implies that $u(G, x+z)<u(0, x)$ and recalling (??), we get that $p(G, x)>z$. Letting $z$ go to $\pi(G)-x$, we obtain that $p(G, x) \geq \pi(G)-x>-\infty$. Applying the preceding inequality to $-G$ and recalling (??) and (??), $p^{B}(G, x) \leq \pi^{s u b}(G)+x<+\infty$. By definition $p(0, x) \leq 0$ and $-x \leq p(0, x)$ follows from $\pi(0)=0$ (see Lemma ??).

2 . The fact that $p(G, x) \geq p(0, x)$ for $G \in \mathcal{W}_{T}^{0,+}$ follows from the monotonicity property that will be proven in Proposition ?? below since the fact that $E_{P} U^{+}\left(\cdot, V_{T}^{x, \phi}(\cdot)\right)<+\infty$, $\forall \phi \in \Phi$ and $\forall P \in \mathcal{Q}^{T}$ implies that $\mathcal{A}(U, G, x)=\mathcal{A}(G, x)$ and $\mathcal{A}(U, 0, x)=\mathcal{A}(0, x)$.

3. We assume now that $u(0, x-\delta)<u(0, x)$ for all $\delta>0$. Then (??) implies that $p(0, x) \geq 0$ and $p(0, x)=p^{B}(0, x)=0$ follows immediately.

\subsection{Risk measures}

We make the link with monetary risk measures introduced in (?), see also (?). Recall that a risk measure allows to quantify by some number $\rho(X)$ a financial position 
described by some $X \in \mathcal{X}$ where $\mathcal{X} \subset \mathcal{W}_{T}^{0}$ is a linear space of random variables (containing the constant random variables) and $X\left(\omega^{T}\right)$ represents the discounted net worth of the position at the end of the trading period if the scenario $\omega^{T} \in \Omega^{T}$ is realized. More precisely,

Definition 2.15 A monetary risk measure is a mapping $\rho: G \in \mathcal{X} \rightarrow \rho(G) \in \mathbb{R} \cup$ $\{ \pm \infty\}$ that verifies

1. for $G, H \in \mathcal{X}$, if $G \geq H \mathcal{Q}^{T}$-q.s., then $\rho(G) \leq \rho(H)$ (monotonicity),

2. if $m \in \mathbb{R}$, then $\rho(G+m)=\rho(G)-m$ (cash invariance).

The measure $\rho$ is said to be a normalized if $\rho(0)=0$ and convex if

3. for all $0 \leq \lambda \leq 1, G, H \in \mathcal{X}, \rho(\lambda G+(1-\lambda) H) \leq \lambda \rho(G)+(1-\lambda) \rho(H)$ (convexity).

We refer to (?, Section 4) (where similar definitions are introduced) for a detailed interpretation of these properties.

We now discuss the relations with the multiple-priors sub- and superreplication prices as well as with the buying and selling prices. From the cash invariance property, a risk-measure can also be seen as a capital requirement: $\rho(G)$ is the amount of cash to be held in addition to the financial instrument $G$ for the aggregate position to be acceptable (from the point of view of a risk-manager, regulator,...). With this in mind, the acceptance set of $\rho$ is often defined by $\{G \in \mathcal{X}, \rho(G) \leq 0\}$. In our context, to measure the risk of a position one can set for some $x \geq 0$

$$
\rho_{x}: G \in \mathcal{X} \rightarrow p(-G, x),
$$

see for example (?, Definition 1.2). We also consider the following measure

$$
\rho: G \in \mathcal{X} \rightarrow \pi(-G) .
$$

Assume for a moment that $\rho_{x}$ and $\rho$ verify the cash invariance property of Definition ??. We consider an agent with initial capital $x$ who is willing to buy and hedge (by trading dynamically in the market) an option whose non-negative payoff is represented by some $G \in \mathcal{X}^{+}$for a price $p_{b}$. Then we have that $\rho_{x}\left(G-p_{b}\right)=\rho_{x}(G)+p_{b}=$ $p(-G, x)+p_{b}=-p^{B}(G, x)+p_{b}$ (see (??) and (??)) and the position is acceptable for the measure $\rho_{x}$ as long as she can buy the contingent claim at or below her buyer multiple-priors utility indifference price $p^{B}(G, x)$. From the point of view of the measure $\rho$, the position is acceptable as soon as $\rho\left(G-p_{b}\right)=\pi(-G)+p_{b}=-\pi^{s u b}(G)+p_{b}$ (see Lemma ??) and the position is acceptable as long as she can buy the contingent claim at or below her multiple-priors subreplication price $\pi^{s u b}(G)$.

Alternatively, if the agent is now considering selling the option at price $p_{s}$ and hedging it, the short position in the contingent claim is represented by $-G \in \mathcal{X}^{-}$and in this case the risk of her position is measured by $\rho_{x}\left(-G+p_{s}\right)=\rho_{x}(-G)-p_{s}=$ $p(G, x)-p_{s}$ : this position will be acceptable if she can sell the option at or above her seller multiple-priors utility indifference price. From the point of view of the measure $\rho$ the position will be acceptable if she can sell the option at or above her multiple-priors superreplication price $\pi(G)$.

Note finally that if $\rho_{x}$ is a normalized convex monetary measure of risk on $\mathcal{W}_{T}^{0}$, we have that $0=\rho_{x}(0) \leq \frac{1}{2}\left(\rho_{x}(G)+\rho_{x}(-G)\right)$. Recalling Definition ??, this implies that $p^{s u b}(G, x) \leq p(G, x)$. Similarly one gets $\pi^{s u b}(G) \leq \pi(G)$ (see Lemma ??).

The two following propositions establish that $\rho$ and $\rho_{x}$ (see (??) and (??)) are normalized convex monetary measures of risk under some well chosen conditions.

Proposition 2.16 If Assumptions ?? and ?? hold true then $\rho$ is a normalized convex monetary measure of risk on $\mathcal{W}_{T}^{0}$. 
Proof. We prove 1. of Definition ??. Fix some $G, H \in \mathcal{W}_{T}^{0}$ such that $G \geq H \mathcal{Q}^{T}$-q.s. From Theorem ?? there exists $\phi \in \Phi$ such that $V_{T}^{\pi(-H), \phi} \geq-H \geq-G \mathcal{Q}^{T}$-q.s. and $\pi(-H) \geq \pi(-G)$. The cash invariance and the convexity are also straightforward and the normalization condition follows from Lemma??

Proposition 2.17 Let $x \geq 0$ be fixed. Assume that $\mathcal{A}(U, G, x)=\mathcal{A}(G, x)$ for all $G \in \mathcal{W}_{T}^{0}$.

1. The mapping $\rho_{x}$ is a monetary measure of risk on $\mathcal{W}_{T}^{0}$.

2. If Assumptions ?? and ?? hold true and $u(0, x)>-\infty$ then $\rho_{x}$ is a convex monetary measure of risk on $\left\{G \in \mathcal{W}_{T}^{0}, u(-G, z)<\infty, \forall z \in \mathbb{R}\right\}$.

3. If furthermore we assume that $u(0, x-\delta)<u(0, x)$ for all $\delta>0$, then $\rho_{x}$ is normalized.

Remark 2.18 It will be clear from the proof that the cash invariance property holds true without the assumption that $\mathcal{A}(U, G, x)=\mathcal{A}(G, x)$ for all $G \in \mathcal{W}_{T}^{0}$. We will give in Proposition ?? and Lemma ?? some conditions under which $\mathcal{A}(U, G, x)=\mathcal{A}(G, x)$ and $u(G, z)<\infty$. Those conditions are needed in order to prove our asymptotic result (see Theorem ??). Note that if we assume that $U$ is bounded from above the two preceding conditions are obviously satisfied.

Proof. See Appendix

\section{Absolute risk aversion and certainty equivalent}

We present now a formal definition of the notion of absolute risk aversion for a general random utility function.

Definition 3.1 For any function $U$ satisfying Definition ??, the absolute risk aversion is defined for all $\left(\omega^{T}, x\right) \in \Omega^{T} \times(0,+\infty)$ by

$$
r\left(\omega^{T}, x\right):=-\frac{U^{\prime \prime}\left(\omega^{T}, x\right)}{U^{\prime}\left(\omega^{T}, x\right)} .
$$

In the mono-prior case, i.e when $\mathcal{Q}^{T}=\{P\}$, the absolute risk aversion is related to the notion of certainty equivalent. If the preferences of an agent are represented by a non-random utility function $U$ and given an asset whose payoff at maturity is $G$, the certainty equivalent $e(G, P)$ is the amount of cash that will make her indifferent (in the sense of the expected utility evaluation) between receiving the cash and the asset $G$

$$
E_{P} U(e(G, P))=U(e(G, P))=E_{P} U(G(\cdot)) .
$$

The risk premium $\rho(G, P):=E_{P} G(\cdot)-e(G, P)$ is the amount that the agent is ready to lose in order to be indifferent (in the sense of the expected utility evaluation) between the sure quantity $E_{P} G(\cdot)-\rho(G, P)$ and the random variable $G$ since

$$
E_{P} U\left(E_{P} G(\cdot)-\rho(G, P)\right)=U(e(G, P))=E_{P} U(G(\cdot)) .
$$

We will see in Proposition ?? that under suitable assumptions $\rho(G, P) \geq 0$. The risk premium is thus a measure of the risk-aversion of the agent: the higher the risk premium, the more risk-adverse the agent is.

The following proposition recalls the definition of the certainty equivalent in a monoprior framework but for random utility functions and proposes an extension to the multiple-priors framework. 
Proposition 3.2 Let $G \in \mathcal{W}_{T}^{0,+}$ such that $G(\cdot)<+\infty \mathcal{Q}^{T}$-q.s.

1. Assume that $U$ is an utility function verifying Definition ??, such that $\sup _{P \in \mathcal{Q}^{T}} E_{P} U^{-}(\cdot, y)<+\infty$ for all $y>0, E_{P} U^{+}(\cdot, 1)<+\infty$ and $E_{P}|U(\cdot, G(\cdot))|<+\infty$ for all $P \in \mathcal{Q}^{T}$.

1.a. For all $P \in \mathcal{Q}^{T}$, there exists a unique constant $e(G, P) \in[0,+\infty)$ such that

$$
E_{P} U(\cdot, e(G, P))=E_{P} U(\cdot, G(\cdot)) .
$$

1.b If furthermore $G \in \mathcal{W}_{T}^{\infty,+}, \sup _{P \in \mathcal{Q}^{T}} E_{P} U^{-}(\cdot, G(\cdot))<\infty$ and $\inf _{P \in \mathcal{Q}^{T}} E_{P} U^{\prime}(\cdot, z)>0$ for all $z>0$, then there exists also an unique $e(G) \in\left[0,\|G\|_{\infty}\right]$ such that

$$
\inf _{P \in \mathcal{Q}^{T}} E_{P} U(\cdot, e(G))=\inf _{P \in \mathcal{Q}^{T}} E_{P} U(\cdot, G(\cdot))
$$

and in this case, we have that $e(G) \geq \inf _{P \in \mathcal{Q}^{T}} e(G, P)$. We call $e(G)$ the multiplepriors certainty equivalent of $G$.

2. Assume now that $U$ is a non-random utility function verifying Definition ?? such that Dom $U=\{x \in \mathbb{R}, U(x)>-\infty\}=(0, \infty), E_{P} U^{+}(G(\cdot))<\infty$ for all $P \in \mathcal{Q}^{T}$ and also $\sup _{P \in \mathcal{Q}^{T}} E_{P} U^{-}(G(\cdot))<\infty$. Then, there exists some unique $e(G, P)$ and $e(G)$ in $[0, \infty)$ such that

$$
\begin{aligned}
U(e(G, P)) & =E_{P} U(G(\cdot)), \forall P \in \mathcal{Q}^{T} \\
U(e(G)) & =\inf _{P \in \mathcal{Q}^{T}} E_{P} U(G(\cdot)) .
\end{aligned}
$$

Moreover, $e(G, P) \leq E_{P} G(\cdot)$ for all $P \in \mathcal{Q}^{T}$ and

$$
e(G)=\inf _{P \in \mathcal{Q}^{T}} e(G, P) \leq \inf _{P \in \mathcal{Q}^{T}} E_{P} G(\cdot) .
$$

Furthermore the multiple-priors risk premium defined by $\rho(G):=\sup _{P \in \mathcal{Q}^{T}} \rho(G, P)$ satisfies

$$
0 \leq \rho(G) \leq \sup _{P \in \mathcal{Q}^{T}} E_{P} G(\cdot)-e(G)
$$

Remark that (??) is true assuming only that $E_{P} U^{-}(G(\cdot))<\infty$ for all $P \in \mathcal{Q}^{T}$.

Proof. See Appendix.

Finally, we consider two investors $A$ and $B$ with respective non-random utility functions $U_{A}$ and $U_{B}$ satisfying Definition ??. Recall that in the mono-prior case with $\mathcal{Q}^{T}=\{P\}$ investor $A$ has greater absolute risk-aversion than investor B (i.e. $r_{A}(x) \geq r_{B}(x)$ for all $x>0$ ) if and only if investor $A$ is globally more risk averse than investor $B$, in the sense that the certainty equivalent of every contingent claim is smaller for $A$ than for $B$ (i.e $e_{A}(G, P) \leq e_{B}(G, P)$ for any $G \in \mathcal{W}_{T}^{0,+}$ ), see (?). We propose the following generalization of this result in the multiple-priors framework.

Proposition 3.3 Let $U_{A}, U_{B}$ be non-random utility functions with domain equal to $(0, \infty)$ verifying Definition ??. Let

$\mathcal{W}_{T}^{+}(U):=\mathcal{W}_{T}^{0,+} \cap\left\{G, G(\cdot)<+\infty \mathcal{Q}^{T}\right.$-q.s., $\left.E_{P} U^{+}(G(\cdot))<\infty, \forall P \in \mathcal{Q}^{T}, \sup _{P \in \mathcal{Q}^{T}} E_{P} U^{-}(G(\cdot))<\infty\right\}$.

1. If for all $x>0, r_{A}(x) \geq r_{B}(x)$ then $e_{A}(G) \leq e_{B}(G)$ for all $G \in \mathcal{W}_{T}^{+}(U)$.

2. If for all $G \in \mathcal{W}_{T}^{+}(U), e_{A}(G)<e_{B}(G)$ then $r_{A}(x) \geq r_{B}(x)$ for all $x>0$. 
Proof. See Appendix.

We prove in Proposition ?? that the absolute risk aversion allows the ranking of the multiple-priors certainty equivalent despite the presence of uncertainty (and thus uncertainty aversion). The reason for this is related to the specific multiplepriors representation we have chosen. For more details we refer to (?, Theorem 5, Example 2).

\section{Convergence of utility indifference prices}

Intuitively speaking an agent who is totally risk averse will use the superreplication price : whatever the possible outcome (where possible outcomes are defined by a set of probability measures), she doesn't want to incur any loss (see (??)). We are going to prove that under suitable assumptions the utility indifference price goes to the superreplication price. For non-random utility functions, the convergence result holds when the absolute risk reversion goes to infinity.

First we give some intuition of this result and show that for a utility function that has a sort of infinite absolute risk aversion, the utility indifference price is equal to the superreplication price for some contingent claim $G \in \mathcal{W}_{T}^{0,+}$. Fix some $x \geq$ $\pi(G)$ and introduce the following utility function $U_{\infty}: \mathbb{R} \rightarrow \mathbb{R} \cup\{-\infty\}$ defined by $U_{\infty}(y)=-\infty 1_{(-\infty, x)}(y)$. Note that the absolute risk aversion of $U_{\infty}$ is not defined. However $U_{n}(y)=-e^{-n(y-x)}$ for $y \geq 0$ and $U_{n}(y)=-\infty$ for $y<0$ satisfies Definition ?? and for $y \geq 0$ fixed with $y \neq x, \lim _{n \rightarrow+\infty} U_{n}(y)=U_{\infty}(y)$. Then the absolute risk aversion of the utility function $U_{n}$ satisfies $\lim _{n \rightarrow+\infty} r_{n}(y)=+\infty$ for all $y \geq 0$. Hence, one may say that $U_{\infty}$ has an infinite absolute risk aversion. We now show that the superreplication price of $G \in \mathcal{W}_{T}^{0,+}$ is equal to its utility indifference price evaluated with the function $U_{\infty}$. Since for all $\phi \in \Phi, y \in \mathbb{R}, U_{\infty}^{+}\left(V_{T}^{y, \phi}(\cdot)-G(\cdot)\right)=0$, we have that $\Phi\left(U_{\infty}, G, y\right)=\Phi\left(U_{\infty}, 0, y\right)=\Phi$ and $\mathcal{A}\left(U_{\infty}, y\right)=\mathcal{A}\left(U_{\infty}, G, y\right)$. Moreover $\mathcal{A}\left(U_{\infty}, G, y\right)$ is not empty for all $y \geq \pi(G)$ (see Theorem ??). First, it is easy to see that $u_{\infty}(0, x)=0$. Now we fix some $0 \leq z<\pi(G)$ and $\phi \in \mathcal{A}\left(U_{\infty}, G, x+z\right)$. There exists some $P \in \mathcal{Q}^{T}$ such that $P\left(V_{T}^{z, \phi}(\cdot)-G(\cdot)<0\right)>0$ or equivalently $P\left(V_{T}^{z+x, \phi}(\cdot)-G(\cdot)<x\right)>0$ which implies that $E_{P} U_{\infty}\left(\cdot, V_{T}^{x+z, \phi}(\cdot)-G(\cdot)\right)=-\infty$. Hence for all $\phi \in \mathcal{A}\left(U_{\infty}, G, x+z\right), \inf _{P \in \mathcal{Q}^{T}} E_{P} U_{\infty}\left(\cdot, V_{T}^{x+z, \phi}(\cdot)-G(\cdot)\right)=-\infty$ and it follows that $u_{\infty}(G, x+z)=-\infty<u_{\infty}(0, x)$. From the definition of $p(G, x)$ we get that $p(G, x) \geq z$ and letting $z$ go to $\pi(G), p(G, x) \geq \pi(G)$. Combining this with Lemma ?? below we have that $p(G, x)=\pi(G)$.

Now we state precisely our convergence result. We consider a sequence of utility functions (see Definition ??) $U_{n}: \Omega^{T} \times \mathbb{R} \rightarrow \mathbb{R} \cup\{-\infty\}, n \geq 1$ and some contingent claim $G \in \mathcal{W}_{T}^{0,+}$. We denote for all $n \geq 1, x \geq 0, \omega^{T} \in \Omega^{T}$

$$
\begin{aligned}
u_{n}(G, x) & :=\sup _{\phi \in \mathcal{A}\left(U_{n}, G, x\right)} \inf _{P \in \mathcal{Q}^{T}} E_{P} U_{n}\left(\cdot, V_{T}^{x, \phi}(\cdot)-G(\cdot)\right) \\
p_{n}(G, x) & :=\inf \left\{z \in \mathbb{R}, u_{n}(G, x+z) \geq u_{n}(0, x)\right\} \\
r_{n}\left(\omega^{T}, x\right) & :=-\frac{U_{n}^{\prime \prime}\left(\omega^{T}, x\right)}{U_{n}^{\prime}\left(\omega^{T}, x\right)} .
\end{aligned}
$$


We review the assumptions needed in Theorem ?? in order to have the convergence result.

Assumption 4.1 We have that $\Delta S_{t}, \frac{1}{\alpha_{t}} \in \mathcal{W}_{t}^{r}$ for all $1 \leq t \leq T$ and $0<r<\infty$ where $\mathcal{W}_{t}^{r}:=\left\{X: \Omega^{t} \rightarrow \mathbb{R} \cup\{ \pm \infty\}, \mathcal{B}_{c}\left(\Omega^{t}\right)\right.$-measurable, $\left.\sup _{P \in \mathcal{Q}^{t}} E_{P}|X|^{r}<\infty\right\}$.

Note that as in (?, Propositions 14 and 15) one can prove that for all $r \in[1, \infty]$, $\mathcal{W}_{t}^{r}$ is a Banach space (up to the usual quotient identifying two random variables that are $\mathcal{Q}^{t}$-q.s. equal) for the norm $\|X\|_{r, t}:=\left(\sup _{P \in \mathcal{Q}^{t}} E_{P}|X|^{r}\right)^{\frac{1}{r}}$ if $r<\infty$ and $\|X\|_{\infty, t}:=\inf \left\{M \geq 0, X(\cdot) \leq M \mathcal{Q}^{t}\right.$ q.s. $\}$. We will omit the index $t$ when $t=T$.

In the light of Proposition ??, the condition $\frac{1}{\alpha_{t}} \in \mathcal{W}_{t}^{r}$ is a kind of strong form of noarbitrage. Note that if $\alpha_{t}$ is not constant, then even in the mono-prior case utility maximisation problem may be ill posed (see Example 3.3 in ?), so an integrability assumption on $\frac{1}{\alpha_{t}}$ looks reasonable. Assumption ?? could be weakened to the existence of the $\mathcal{W}_{T}^{N}$-th moment for $N$ large enough but this would lead to complicated book-keeping with no essential gain in generality, which we prefer to avoid.

The asymptotic result for general random utility functions will be stated for some fixed $x_{0}>0$. However in the case of non-random utility functions we can avoid Assumption ?? below and obtain the convergence result for all $x>0$. We can also use the natural assumption that $\lim _{n \rightarrow+\infty} r_{n}(x)=+\infty$ instead of Assumption ??, see Theorem ??.

The first assumption states that $U_{n}$ is sufficiently measurable and regular in $x_{0}$.

Assumption 4.2 We have that $\sup _{n}\left\|U_{n}^{ \pm}\left(\cdot, x_{0}\right)\right\|_{1}<\infty$ and that $\sup _{n}\left\|U_{n}^{\prime}\left(\cdot, x_{0}\right)\right\|_{q}<\infty$ for some $q>1$.

Remark 4.3 If we assume that $\sup _{n} U_{n}^{ \pm}\left(\cdot, x_{0}\right) \in \mathcal{W}_{T}^{1}$ and that there exists some $q>1$ such that $\sup _{n}\left\|U_{n}^{\prime}\left(\cdot, x_{0}\right)\right\|_{q}<\infty$, then Assumption ?? is verified. Indeed let's prove for instance that $\sup _{n}\left\|E_{P} U_{n}^{+}\left(\cdot, x_{0}\right)\right\|_{1}<+\infty$. For all $P \in \mathcal{Q}^{T}, n \geq 1$ we have that $E_{P} U_{n}^{+}\left(\cdot, x_{0}\right) \leq E_{P} \sup _{n} U_{n}^{+}\left(\cdot, x_{0}\right)$ and it follows that $\sup _{n} \sup _{P \in \mathcal{Q}^{T}} E_{P} U_{n}^{+}\left(\cdot, x_{0}\right) \leq\left\|\sup _{n} U_{n}^{+}\left(\cdot, x_{0}\right)\right\|_{1}$.

We postulate now the assumption which will play the role of the convergence of the absolute risk aversion to infinity for random utility function.

Assumption 4.4 For all $0 \leq x<x_{0}$ and $M \geq 0$,

$$
\lim _{n \rightarrow+\infty} \inf _{P \in \mathcal{Q}^{T}} P\left(U_{n}(\cdot, x) \leq-M\right)=1 .
$$

Assumption ?? means that $U_{n}(\cdot, x)$ goes to $-\infty$ with respect to $\inf _{P \in \mathcal{Q}^{T}} P$ for all $0 \leq$ $x<x_{0}$. We propose a first lemma with an alternative condition to (??).

Lemma 4.5 Suppose that for all $n \geq 1, U_{n}$ verifies Assumption ?? and that for all $\varepsilon>0$ such that $x_{0}>\varepsilon$ and all $C \geq 0$

$$
\lim _{n \rightarrow \infty} \inf _{P \in \mathcal{Q}^{T}} P\left(\left\{\int_{x_{0}-\frac{\varepsilon}{2}}^{x_{0}} U_{n}^{\prime \prime}(\cdot, v) d v<-\frac{C}{\varepsilon}\right\}\right)=1 .
$$

Then Assumption ?? holds true. 
Proof. Fix some $\varepsilon>0$ such that $x_{0}>\varepsilon$ and $M \geq 0$. For all $\omega^{T} \in \Omega^{T} U_{n}\left(\omega^{T}, x_{0}-\right.$ $\varepsilon)=U_{n}\left(\omega^{T}, x_{0}\right)-\int_{x_{0}-\varepsilon}^{x_{0}} U_{n}^{\prime}\left(\omega^{T}, u\right) d u$. Using that $U_{n}^{\prime}\left(\omega^{T}, \cdot\right)$ is non-negative and non decreasing (see Definition ??), we obtain that

$U_{n}\left(\omega^{T}, x_{0}-\varepsilon\right)+\frac{\varepsilon}{2} U_{n}^{\prime}\left(\omega^{T}, x_{0}-\frac{\varepsilon}{2}\right) \leq U_{n}\left(\omega^{T}, x_{0}-\varepsilon\right)+\int_{x_{0}-\varepsilon}^{x_{0}-\frac{\varepsilon}{2}} U_{n}^{\prime}\left(\omega^{T}, v\right) d v \leq U_{n}\left(\omega^{T}, x_{0}\right)$.

Now

$$
U_{n}^{\prime}\left(\omega^{T}, x_{0}-\frac{\varepsilon}{2}\right)=U_{n}^{\prime}\left(\omega^{T}, x_{0}\right)-\int_{x_{0}-\frac{\varepsilon}{2}}^{x_{0}} U_{n}^{\prime \prime}\left(\omega^{T}, v\right) d v \geq-\int_{x_{0}-\frac{\varepsilon}{2}}^{x_{0}} U_{n}^{\prime \prime}\left(\omega^{T}, v\right) d v
$$

and all together

$$
U_{n}\left(\omega^{T}, x_{0}-\varepsilon\right) \leq\left|U_{n}\left(\omega^{T}, x_{0}\right)\right|+\frac{\varepsilon}{2} \int_{x_{0}-\frac{\varepsilon}{2}}^{x_{0}} U_{n}^{\prime \prime}\left(\omega^{T}, v\right) d v .
$$

We fix some $\eta>0$ and show that there exists some $N_{\eta}>0$ such that $\inf _{P \in \mathcal{Q}^{T}} P\left(\left|U_{n}\left(\cdot, x_{0}\right)\right| \leq N_{\eta}\right)>$ $1-\frac{\eta}{2}$ for all $n$. Indeed using (?, Lemma 13) and Assumption ?? we get that

$$
\sup _{P \in \mathcal{Q}^{T}} P\left(\left|U_{n}\left(\cdot, x_{0}\right)\right|>k\right) \leq \frac{1}{k} \sup _{P \in \mathcal{Q}^{T}} E_{P}\left(\left|U_{n}\left(\cdot, x_{0}\right)\right|\right) \leq \frac{1}{k} \sup _{n}\left\|U_{n}\left(\cdot, x_{0}\right)\right\|_{1} .
$$

Thus there exists $N_{\eta}>0$ such that $\sup _{P \in \mathcal{Q}^{T}} P\left(\left|U_{n}\left(\cdot, x_{0}\right)\right|>N_{\eta}\right)<\frac{\eta}{2}$ for all $n$. From (??) with $C=2\left(N_{\eta}+M\right)$, there exists $N=N(\eta, M, \varepsilon)$ such that for all $n \geq N$,

$$
\begin{array}{rl}
\inf _{P \in \mathcal{Q}^{T}} & P\left(U_{n}\left(\cdot, x_{0}-\varepsilon\right) \leq-M\right) \\
& \geq \inf _{P \in \mathcal{Q}^{T}} P\left(\left\{\left|U_{n}\left(\cdot, x_{0}\right)\right| \leq N_{\eta}\right\} \cap\left\{\int_{x_{0}-\frac{\varepsilon}{2}}^{x_{0}} U_{n}^{\prime \prime}\left(\omega^{T}, v\right) d v<-\frac{2\left(N_{\eta}+M\right)}{\varepsilon}\right\}\right) \\
& \geq \inf _{P \in \mathcal{Q}^{T}} P\left(\left\{\left|U_{n}\left(\cdot, x_{0}\right)\right| \leq N_{\eta}\right\}\right)+\inf _{P \in \mathcal{Q}^{T}} P\left(\left\{\int_{x_{0}-\frac{\varepsilon}{2}}^{x_{0}} U_{n}^{\prime \prime}\left(\omega^{T}, v\right) d v<-\frac{2\left(N_{\eta}+M\right)}{\varepsilon}\right\}\right)-1 \\
& >1-\eta .
\end{array}
$$

Thus, (??) is proved for all $x=x_{0}-\varepsilon>0$. Since $U_{n}$ is (strictly) increasing (??) is also true for $x=0$ and this concludes the proof.

We provide another lemma which gives sets of assumptions under which (??) (and thus Assumption ?? under Assumption ??) is satisfied. It is stated under the assumption that $U_{n}$ is strictly increasing in $x_{0}$ uniformly in $n$.

Lemma 4.6 Let $\varepsilon>0$ such that $x_{0}>\varepsilon$ and $C \geq 0$. Assume that there exists a strictly positive random variable $\lambda$ such that $U_{n}^{\prime}\left(\omega^{T}, x_{0}\right) \geq \lambda\left(\omega^{T}\right)$ for all $\omega^{T} \in \Omega^{T}, n \geq 1$ and that either 1., 2. or 3. below are satisfied. Then (??) is verified.

1. For all $n$ and $\omega^{T} \in \Omega^{T}, U_{n}^{\prime \prime}\left(\omega^{T}, \cdot\right)$ is non decreasing, and

$$
\lim _{n \rightarrow \infty} \inf _{P \in \mathcal{Q}^{T}} P\left(\left\{\lambda(\cdot) r_{n}\left(\cdot, x_{0}\right)>\frac{2 C}{\varepsilon^{2}}\right\}\right)=1 .
$$

2. We have that

$$
\lim _{n \rightarrow \infty} \inf _{P \in \mathcal{Q}^{T}} P\left(\lambda(\cdot) \int_{x_{0}-\frac{\varepsilon}{2}}^{x_{0}} r_{n}(\cdot, v) d v>\frac{C}{\varepsilon}\right)=1 .
$$

3. There exists some deterministic functions $\left(r_{n}\right)_{n \geq 1}$ satisfying $\lim _{n} r_{n}(x)=+\infty$ and $r_{n}\left(\omega^{T}, x\right) \geq r_{n}(x)$ for all $x \in\left(0, x_{0}\right]$. 
Remark 4.7 1. If we only assume that $\lim _{n \rightarrow+\infty} r_{n}\left(\omega^{T}, x\right)=+\infty$ for all $x \in\left(0, x_{0}\right]$, $\omega^{T} \in \Omega^{T}$ we cannot deduce a priori 2 . Indeed applying Fatou's Lemma we get that for all $\omega^{T} \in \Omega^{T}$, there exists $N_{\omega^{T}}$ such that for all $k \geq N_{\omega^{T}}, \lambda\left(\omega^{T}\right) \int_{x_{0}-\frac{\varepsilon}{2}}^{x_{0}} r_{n}\left(\omega^{T}, v\right) d v>\frac{C}{\varepsilon}$, which means that

$$
\Omega^{T}=\cup_{n} \cap_{k \geq n}\left\{\lambda(\cdot) \int_{x_{0}-\frac{\varepsilon}{2}}^{x_{0}} r_{k}(\cdot, v) d v>\frac{C}{\varepsilon}\right\}
$$

and using (?, Theorem 1) this implies that

$$
\lim _{n \rightarrow \infty} \sup _{P \in \mathcal{Q}^{T}} P\left(\lambda(\cdot) \int_{x_{0}-\frac{\varepsilon}{2}}^{x_{0}} r_{n}(\cdot, v) d v>\frac{C}{\varepsilon}\right)=1 .
$$

2. For assertion 1. that power utility functions or exponential utility functions (with random coefficients, see Example ?? for the precise conditions) are examples where $U_{n}^{\prime \prime}\left(\omega^{T}, \cdot\right)$ is non decreasing for all $n$ and $\omega^{T} \in \Omega^{T}$.

3. Note lastly that it is easy to see that Definition ?? $i i)$ or $\lambda>0$ or $U_{n}$ strictly increasing in $x_{0}$ uniformly in $n$ can be postulate only on a $\mathcal{Q}^{T}$-full measure set.

Proof of Lemma ??. We start with 1. Since for all $n$ and $\omega^{T} \in \Omega^{T}, U_{n}^{\prime \prime}\left(\omega^{T}, \cdot\right)$ is non decreasing and $U_{n}^{\prime}\left(\omega^{T}, x_{0}\right) \geq \lambda\left(\omega^{T}\right)$, we get that

$$
\int_{x_{0}-\frac{\varepsilon}{2}}^{x_{0}} U_{n}^{\prime \prime}(\cdot, v) d v \leq \frac{\varepsilon}{2} U_{n}^{\prime \prime}\left(\cdot, x_{0}\right)=-\frac{\varepsilon}{2} U_{n}^{\prime}\left(\cdot, x_{0}\right) r_{n}\left(\cdot, x_{0}\right) \leq-\frac{\varepsilon}{2} \lambda(\cdot) r_{n}\left(\cdot, x_{0}\right) .
$$

Thus (??) implies (??). For 2 . since for all $n$ and $\omega^{T} \in \Omega^{T}, U_{n}^{\prime}\left(\omega^{T}, x_{0}\right) \geq \lambda\left(\omega^{T}\right)$, we get that

$$
\int_{x_{0}-\frac{\varepsilon}{2}}^{x_{0}} U_{n}^{\prime \prime}(\cdot, v) d v=-\int_{x_{0}-\frac{\varepsilon}{2}}^{x_{0}} U_{n}^{\prime}(\cdot, v) r_{n}(\cdot, v) d v \leq-\lambda(\cdot) \int_{x_{0}-\frac{\varepsilon}{2}}^{x_{0}} r_{n}(\cdot, v) d v .
$$

Thus (??) implies (??). For 3. we prove that 2. holds true. Indeed Fatou's Lemma implies that $\lim _{n \rightarrow \infty} \int_{x_{0}-\frac{\varepsilon}{2}}^{x_{0}} r_{n}(v) d v=+\infty$ and we conclude since $\left\{\lambda(\cdot) \int_{x_{0}-\frac{\varepsilon}{2}}^{x_{0}} r_{n}(v) d v>\right.$ $\left.\frac{C}{\varepsilon}\right\} \subset\left\{\lambda(\cdot) \int_{x_{0}-\frac{\varepsilon}{2}}^{x_{0}} r_{n}(\cdot, v) d v>\frac{C}{\varepsilon}\right\}$.

Example 4.8 We give a concrete example of some random utility function satisfying Definition ?? and Assumptions ?? and ??. For all $n \geq 1$, let $R_{n}$ be a random variable uniformly distributed in $\left[b_{n}, B_{n}\right]$ for all $P \in \mathcal{Q}^{T}$ with $b_{n}>0, \lim _{n \rightarrow \infty} b_{n}=+\infty$ and $B_{n}^{3}-b_{n}^{3}<A$ for some $A>0$. Note that as $b_{n}>0$ and $\lim _{n \rightarrow \infty} b_{n}=+\infty$, there exists some $b>0$ such that $b_{n} \geq b$ for all $n \geq 1$. Set now $U_{n}\left(\omega^{T}, x\right)=-e^{-R_{n}\left(\omega^{T}\right)(x-1)}$ for $x \geq 0$ and $U_{n}\left(\omega^{T}, x\right)=-\infty$ for $x<0$. We choose $x_{0}=1$. As $U_{n}^{\prime}(\cdot, 1)=R_{n}(\cdot) \geq b>0$ $\mathcal{Q}^{T}$ q.s., $U_{n}(\cdot, 1)$ is uniformly increasing in $n$ and $\omega^{T}$. Now $\left\|U_{n}^{\prime}(\cdot, 1)\right\|_{2}^{2}=\frac{B_{n}^{3}-b_{n}^{3}}{3}$, thus $\sup _{n}\left\|U_{n}^{\prime}(\cdot, 1)\right\|_{2}<\infty$. Then as $U_{n}(\cdot, 1)=-1$, Assumption ?? holds true. Finally for all $n \geq 1, x>0$ and $\omega^{T} \in \Omega^{T}$ we have $r_{n}\left(\omega^{T}, x\right)=R_{n}\left(\omega^{T}\right) \geq b_{n}$ and $\lim _{n} b_{n}=+\infty$, so using Lemmata ?? and ??, Assumption ?? is verified.

Theorem 4.9 Let $G \in \mathcal{W}_{T}^{0,+}$ and $G \neq 0 \mathcal{Q}^{T}$-q.s. Assume that Assumptions ??, ??, ?? and ?? holds true as well as Assumptions ?? and ?? for some $x_{0}>0$. For all $n \geq 1$, $p_{n}\left(G, x_{0}\right)$ is well defined and $\lim _{n \rightarrow+\infty} p_{n}\left(G, x_{0}\right)=\pi(G)$.

If $G=0 \mathcal{Q}^{T}$-q.s. then $\pi(G)=0$ (see Lemma ??) but in order to have that $p_{n}\left(G, x_{0}\right)=0$, one have to make further assumptions (see Proposition ??). 
Theorem 4.10 Let $G \in \mathcal{W}_{T}^{0,+} G \neq 0 \mathcal{Q}^{T}$-q.s. Assume that Assumptions ??, ??, ??, ?? hold true. Assume furthermore that $U_{n}$ is a non-random utility function for all $n \geq 1$ and that $\lim _{n \rightarrow \infty} r_{n}(x)=+\infty$. Then $\lim _{n \rightarrow+\infty} p_{n}(G, x)=\pi(G)$ for all $x>0$.

Proof of Theorem ??. We fix some $x>0$. As in (?), we replace $U_{n}$ by $\hat{U}_{n}:=\alpha_{n} U_{n}+\beta_{n}$ for some $\alpha_{n}>0$ and $\beta_{n} \in \mathbb{R}, \hat{U}_{n}$ is still a non-random, concave, strictly increasing and twice continuously differentiable function. The absolute risk aversion and the utility indifference price for $U_{n}$ and $\hat{U}_{n}$ (see (??)) are the same. Now, choosing $\alpha_{n}=\frac{1}{U_{n}^{\prime}(x)}$ and $\beta_{n}=-\frac{U_{n}(x)}{U_{n}^{\prime}(x)}$ we have that $\hat{U}_{n}(x)=0$ and $\hat{U}_{n}^{\prime}(x)=1$ for all $n \geq 1$. Thus $\hat{U}_{n}$ satisfies Assumptions ?? for $x_{0}=x$. Using Lemmata ?? and ??, Assumption ?? for $\hat{U}_{n}$ holds true for $x_{0}=x$ and Theorem ?? applies to $\hat{U}_{n}$.

Recalling the definition of the subreplication price (see (??)) and the buyer multiplepriors utility indifference price (see (??)), the following proposition is a simple consequence of Theorem ??.

Proposition 4.11 Let $G \in \mathcal{W}_{T}^{\infty,+}$ such that $G \neq 0 \mathcal{Q}^{T}$-q.s. Assume that Assumptions ??, ??, ?? and ?? holds true as well as Assumptions ?? and ?? for some $x_{0}>0$. Then, for all $n \geq 1, p_{n}^{B}\left(G, x_{0}\right)$ is well defined and $\lim _{n \rightarrow+\infty} p_{n}^{B}\left(G, x_{0}\right)=\pi^{\text {sub }}(G)$.

Proof of Proposition ??. Let $\widehat{G}=-G+\|G\|_{\infty} \in \mathcal{W}_{T}^{0,+}$. We apply Theorem ?? to $\widehat{G}$ and get that $\lim _{n \rightarrow \infty} p_{n}\left(\widehat{G}, x_{0}\right)=\pi(\widehat{G})$. From the cash invariance property in Proposition ??, we have that $\pi(\widehat{G})=\pi(-G)+\|G\|_{\infty}=-\pi^{s u b}(G)+\|G\|_{\infty}$, see Lemma ??. Now for $n \geq 1$, using the cash invariance property in Proposition ?? and recalling (??) we obtain that $p_{n}\left(\widehat{G}, x_{0}\right)=p_{n}\left(-G, x_{0}\right)+\|G\|_{\infty}=-p_{n}^{B}\left(G, x_{0}\right)+\|G\|_{\infty}$ and the result follows.

Recalling the definition of the risk measures $\rho_{x_{0}}^{n}$ and $\rho$ (see (??) and (??)), the fact that $p^{B}(G, x)=-p(-G, x)$ and $\pi^{s u b}(G)=-\pi(-G)$ (see Lemma ??), Proposition ?? can be reformulated in terms of risk measures.

Proposition 4.12 Assume that Assumptions ??, ??, ?? and ?? holds true as well as Assumptions ?? and ?? for some $x_{0}>0$. Then $\lim _{n \rightarrow+\infty} \rho_{x_{0}}^{n}(G)=\rho(G)$ for all $G \in \mathcal{W}_{T}^{\infty,+}$ such that $G \neq 0 \mathcal{Q}^{T}$-q.s.

The following proposition shows that whatever the strategy is, the wealth is uniformly bounded.

Proposition 4.13 Fix some $x \geq 0$. Assume that Assumptions ??, ??, ??, ?? and ?? hold true. Then for all $\phi \in \mathcal{A}(0, x)$ and for all $1 \leq t \leq T$, we have for $\mathcal{Q}^{t}$-q.s. all $\omega^{t} \in \Omega^{t}$ that

$$
\left|V_{t}^{x, \phi}\left(\omega^{t}\right)\right| \leq x \prod_{s=1}^{t}\left(1+\frac{\left|\Delta S_{s}\left(\omega^{s}\right)\right|}{\alpha_{s-1}\left(\omega^{s-1}\right)}\right):=x M_{t}\left(\omega^{t}\right)
$$

where $M_{1}=1$. Furthermore for all $1 \leq t \leq T$, we have that $M_{t} \geq 1$, that $M_{t}, V_{t}^{x, \phi} \in$ $\mathcal{W}_{t}^{r}$ for all $r \in[0, \infty)$ and that for all $P \in \mathcal{Q}^{T}$ and $n \geq 1$

$$
E_{P} U_{n}^{+}\left(\cdot, V_{T}^{x, \phi}(\cdot)\right) \leq K_{x},
$$

where $K_{x}:=\sup _{n}\left\|U_{n}^{+}\left(\cdot, x_{0}\right)\right\|_{1}+x\left\|M_{T}(\cdot)\right\|_{p} \sup _{n}\left\|U_{n}^{\prime}\left(\cdot, x_{0}\right)\right\|_{q}<\infty$ and where $q$ is defined in Assumption ?? and $p$ is such that $\frac{1}{p}+\frac{1}{q}=1$. 
Proof. See Appendix.

Lemma 4.14 Assume that Assumptions ?? and ?? hold true. Fix some $G \in \mathcal{W}_{T}^{0}, x \geq 0$ and some random utility function $U$ verifying Definition ??.

1. Assume that $\mathcal{A}(U, G, \pi(G)+x)=\mathcal{A}(G, \pi(G)+x)$. Then $p(G, x) \leq \pi(G)$.

2. Assume that $\mathcal{A}(U,-G, \pi(-G)+x)=\mathcal{A}(-G, \pi(-G)+x)$. Then $\pi^{s u b}(G) \leq p^{B}(G, x)$.

3. Assume that Assumptions ?? and Assumption ?? stated for $x$ hold true and that $G \in \mathcal{W}_{T}^{0,+}$. Then for all $n \geq 1, \mathcal{A}\left(U_{n}, G, x\right)=\mathcal{A}(G, x)$ and $u_{n}(G, x)<\infty$.

Proof. 1. We apply Theorem ?? and obtain some $\phi_{G} \in \mathcal{A}(G, \pi(G))$. As $U$ is nondecreasing we have that

$$
\begin{aligned}
u(0, x)=\sup _{\phi \in \mathcal{A}(U, 0, x)} \inf _{P \in \mathcal{Q}^{T}} E_{P} U\left(\cdot, V_{T}^{x, \phi}(\cdot)\right) & \leq \sup _{\phi \in \mathcal{A}(U, 0, x)} \inf _{P \in \mathcal{Q}^{T}} E_{P} U\left(\cdot, V_{T}^{x+\pi(G), \phi+\phi_{G}}(\cdot)-G(\cdot)\right) \\
& \leq \sup _{\phi \in \mathcal{A}(U, G, x+\pi(G))} \inf _{P \in \mathcal{Q}^{T}} E_{P} U\left(\cdot, V_{T}^{x+\pi(G), \phi}(\cdot)-G(\cdot)\right) \\
& =u(G, x+\pi(G)),
\end{aligned}
$$

where the second inequality follows from the fact that when $\phi \in \mathcal{A}(U, 0, x) \subset \mathcal{A}(0, x)$, $\phi+\phi_{G} \in \mathcal{A}(G, \pi(G)+x)=\mathcal{A}(U, G, \pi(G)+x)$ by assumption. So $p(G, x) \leq \pi(G)$ follows from (??).

2. Now if $\mathcal{A}(U,-G, \pi(-G)+x)=\mathcal{A}(-G, \pi(-G)+x)$, we obtain that $p(-G, x) \leq \pi(-G)$ and recalling (??) and (??), we get that $\pi^{\text {sub }}(G) \leq p^{B}(G, x)$.

3. If $\mathcal{A}(G, x)=\emptyset$ then $\mathcal{A}\left(U_{n}, G, x\right)=\emptyset$ and $u_{n}(G, x)=-\infty<\infty$ for all $n \geq 1$. We assume now that $\mathcal{A}(G, x) \neq \emptyset$. For all $n \geq 1$, using the monotonicity of $U_{n}$, the fact that $G \geq 0 \mathcal{Q}^{T}$-q.s., Proposition ?? (see (??)), we get that for any $\phi \in \mathcal{A}(G, x)$ and $P \in \mathcal{Q}^{T}$

$$
E_{P} U_{n}^{+}\left(\cdot, V_{T}^{x, \phi}(\cdot)-G(\cdot)\right) \leq E_{P} U_{n}^{+}\left(\cdot, V_{T}^{x, \phi}(\cdot)\right) \leq K_{x}<\infty
$$

Hence the integrals in (??) are well defined and we get that $\mathcal{A}\left(U_{n}, G, x\right)=\mathcal{A}(G, x)$. The fact that $u_{n}(G, x)<\infty$ for all $n \geq 1$ follows immediately from (??).

To prove Theorem ?? we borrow some ideas of (?) adapted to the multiple-priors case. Proof of Theorem ??. Since $G \in \mathcal{W}_{T}^{0,+}$ is such that $G \neq 0 \mathcal{Q}^{T}$-q.s., $\pi(G)>0$ (see Lemma ?? and the monotonicity property in Proposition ??). From Assumption ??, $\sup _{n}\left\|U_{n}^{+}\left(\cdot, x_{0}\right)\right\|_{1}<\infty$, so that $0 \in \mathcal{A}\left(U_{n}, 0, x_{0}\right)$ (recall that $x_{0}>0$ ) for all $n \geq 1$. This implies that for all $n \geq 1$

$$
u_{n}\left(0, x_{0}\right) \geq \inf _{P \in \mathcal{Q}^{T}} E_{P} U_{n}\left(\cdot, x_{0}\right) \geq-\sup _{n}\left\|U_{n}^{-}\left(\cdot, x_{0}\right)\right\|_{1}>-\infty
$$

using Assumption ??.

We treat first the case $\pi(G)=+\infty$. By definition for all $x \in \mathbb{R}, n \geq 1, \emptyset=\mathcal{A}(G, x)=$ $\mathcal{A}\left(U_{n}, G, x\right)$ and recalling (??), $u_{n}\left(G, x_{0}+z\right)=-\infty$ for all $z \in \mathbb{R}$. Recalling (??) and (??) we get that $p_{n}\left(G, x_{0}\right)=+\infty$ for all $n \geq 1$.

We assume now that $\pi(G)<\infty$. Using Lemma ?? we have that $p_{n}\left(G, x_{0}\right) \leq \pi(G)<\infty$ for all $n \geq 1$. Thus, to prove that $\lim _{n \rightarrow \infty} p_{n}\left(G, x_{0}\right)=\pi(G)$ it is enough to show that $\liminf { }_{n} p_{n}\left(G, x_{0}\right) \geq \pi(G)$. Assume that this is not the case. Hence we can find a subsequence $\left(n_{k}\right)_{k \geq 1}$ and some $\eta>0$ such that $p_{n_{k}}\left(G, x_{0}\right) \leq \pi(G)-\eta$ for all $k \geq 1$. Since $x_{0}>0$ and $\pi(G)>0$, we may and will assume that $\eta<\min \left(\pi(G), x_{0}\right)$. By definition of $p_{n_{k}}\left(G, x_{0}\right)$ we have that

$$
u_{n_{k}}\left(G, x_{0}+\pi(G)-\eta\right) \geq u_{n_{k}}\left(0, x_{0}\right) .
$$


Assume that $\lim _{k \rightarrow+\infty} u_{n_{k}}\left(G, x_{0}+\pi(G)-\eta\right)=-\infty$ is proved then $\liminf _{k \rightarrow+\infty} u_{n_{k}}\left(0, x_{0}\right)=$ $-\infty$. But using Assumption ?? (see (??)) $\liminf _{k \rightarrow+\infty} u_{n_{k}}\left(0, x_{0}\right)>-\infty$, a contradiction. Thus, it remains to prove that $\lim _{k \rightarrow+\infty} u_{n_{k}}(G, y)=-\infty$ with $y=x_{0}+\pi(G)-\eta<$ $x_{0}+\pi(G)$. For ease of notation, we will prove that $\lim _{n \rightarrow+\infty} u_{n}(G, y)=-\infty$.

First we show that $x_{0}+G \notin \mathcal{C}_{y}^{T}$ (see (??) for the definition of $\mathcal{C}_{y}^{T}$ ). Indeed if this is not the case, there exists some $X \in \mathcal{W}_{T}^{0,+}$ and $\phi \in \Phi$ such that $x_{0}+G=V_{T}^{y, \phi}-X \mathcal{Q}^{T}$-q.s., hence $G \leq V_{T}^{y-x_{0}, \phi} \mathcal{Q}^{T}$-q.s. Therefore we must have $y-x_{0} \geq \pi(G)$ : a contradiction. Applying Lemma ??, we get some $\varepsilon>0$ such that $\inf _{\phi \in \Phi} \sup _{P \in \mathcal{Q}^{T}} P\left(A_{\phi}\right)>\varepsilon$, where $A_{\phi}:=\left\{V_{T}^{y, \phi}(\cdot)<x_{0}+G(\cdot)-\varepsilon\right\}$. Note that we can always assume that $x_{0} \geq \varepsilon$. Hence for all $\phi \in \Phi$, there exists some $P_{\varepsilon, \phi} \in \mathcal{Q}^{T}$ such that $P_{\varepsilon, \phi}\left(A_{\phi}\right)>\varepsilon$. From Lemma ?? and Theorem ??, we get that $\mathcal{A}\left(U_{n}, G, y\right)=\mathcal{A}(G, y) \neq \emptyset$ since $y \geq \pi(G)$. We choose some $\phi \in \mathcal{A}(G, y)$. Using the monotonicity of $U_{n}$ and recalling (??) (since $G(\cdot) \geq 0 \mathcal{Q}^{T}$-q.s.), we get that

$$
E_{P_{\varepsilon, \phi}} 1_{\Omega^{T} \backslash A_{\phi}} U_{n}\left(\cdot, V_{T}^{y, \phi}(\cdot)-G(\cdot)\right) \leq E_{P_{\varepsilon, \phi}} U_{n}^{+}\left(\cdot, V_{T}^{y, \phi}(\cdot)\right) \leq K_{y} \leq K_{x_{0}+\pi(G)} .
$$

Fix some $J>0$ and set $C_{J}:=\frac{2}{\varepsilon}\left(J+K_{x_{0}}+K_{x_{0}+\pi(G)}\right)$ and $B_{J, n}:=\left\{U_{n}\left(\cdot, x_{0}-\varepsilon\right) \leq\right.$ $\left.-C_{J}\right\}$. We apply Assumption ?? and obtain that there exists some $N_{J} \geq 1$ (which does not depend on $\phi$ ) such that for all $n \geq N_{J}$,

$$
P_{\varepsilon, \phi}\left(B_{J, n}\right) \geq \inf _{P \in \mathcal{Q}^{T}} P\left(B_{J, n}\right)>1-\frac{\varepsilon}{2} .
$$

It follows that for all $n \geq N_{J}, P_{\varepsilon, \phi}\left(B_{J, n} \cap A_{\phi}\right)>\frac{\varepsilon}{2}$ and we get that

$$
\begin{aligned}
E_{P_{\varepsilon, \phi}} 1_{A_{\phi}} U_{n}\left(\cdot, V_{T}^{y, \phi}(\cdot)-G(\cdot)\right) & \leq E_{P_{\varepsilon, \phi}} 1_{A_{\phi} \cap B_{J, n}} U_{n}\left(\cdot, x_{0}-\varepsilon\right)+E_{P_{\varepsilon, \phi}} 1_{A_{\phi} \backslash B_{J, n}} U_{n}\left(\cdot, x_{0}\right) \\
& \leq \frac{-\varepsilon C_{J}}{2}+K_{x_{0}}=-J-K_{x_{0}+\pi(G)},
\end{aligned}
$$

using (??) and the definition of $C_{J}$. Combining the previous equation with (??), we obtain for all $n \geq N_{J}$ that

$$
\inf _{P \in \mathcal{Q}^{T}} E_{P} U_{n}\left(\cdot, V_{T}^{y, \phi}(\cdot)-G(\cdot)\right) \leq E_{P_{\varepsilon, \phi}} U_{n}\left(\cdot, V_{T}^{y, \phi}(\cdot)-G(\cdot)\right) \leq-J .
$$

As $N_{J}$ doesn't depend on $\phi$, recalling the definition of $u_{n}$ (see (??)), we obtain for all that $n \geq N_{J}, u_{n}(y, G) \leq-J$. Since this is true for all $J \geq 0, \lim _{n \rightarrow \infty} u_{n}(G, y)=-\infty$ and the proof is complete.

\section{Appendix}

Proof of Proposition ??. We prove 1. of Definition ??. Fix some $G, H \in \mathcal{W}_{T}^{0}$ such that $G \geq H \mathcal{Q}^{T}$-q.s. As $\mathcal{A}(U,-H, x)=\mathcal{A}(-H, x) \subset \mathcal{A}(-G, x)=\mathcal{A}(U,-G, x)$, it is easy to check that for all $z \in \mathbb{R}, u(-H, x+z) \leq u(-G, x+z)$ (recall that $U$ is increasing, see Definition ??). If $\rho_{x}(H)=+\infty$, there is nothing to prove, while if $\rho_{x}(H)=-\infty$, it is clear that $\rho_{x}(G)=-\infty$ also. Assume now that $\rho_{x}(H)$ is finite and fix some $\varepsilon>0$. Then $u(0, x) \leq u(-H, x+p(-H, x)+\varepsilon) \leq u(-G, x+p(-H, x)+\varepsilon)$ and we get that $p(-G, x) \leq p(-H, x)+\varepsilon$. As this is true for all $\varepsilon, \rho_{x}(G) \leq \rho_{x}(H)$ follows immediately. We prove 2. of Definition ??. We fix some $m \in \mathbb{R}$. It is clear (without the assumption that $\mathcal{A}(U, G, x)=\mathcal{A}(G, x)$ for all $\left.G \in \mathcal{W}_{T}^{0}\right)$ that $\mathcal{A}(U,-(G+m), x)=\mathcal{A}(U,-G, x+m)$ and 
it follows that $u(-(G+m), x)=u(-G, x+m)$. One can easily see that $\rho_{x}(G+m, x)=$ $+\infty$ (resp. $=-\infty)$ if and only if $\rho_{x}(G, x)=+\infty$ (resp. $\left.=-\infty\right)$. So we may assume that $\rho_{x}(G+m, x)$ is finite and fix some $\varepsilon>0$. Then

$$
u(0, x) \leq u(-(G+m), x+p(-(G+m), x)+\varepsilon)=u(-G, x+p(-(G+m), x)+m+\varepsilon) .
$$

Thus $p(-G, x) \leq p(-(G+m), x)+m+\varepsilon$ follows. We have also that

$$
u(0, x) \leq u(-G, x+p(-G, x)+\varepsilon)=u(-(G+m), x-m+p(-G, x)+\varepsilon)
$$

and $p(-(G+m), x) \leq p(-G, x)-m+\varepsilon$. Letting $\varepsilon$ go to 0 , we get that $p(-G, x)-m=$ $p(-(G+m), x)$ as claimed.

We prove now the second part of the proposition. From Proposition ??, one gets that $p(-G, x)>-\infty$ and $p(-H, x)>-\infty$ for some fixed $G, H \in\left\{X \in \mathcal{W}_{T}^{0}, u(-X, z)<\right.$ $\infty, \forall z \in \mathbb{R}\}$. We want to show that for all $0<\lambda<1$

$$
\lambda p(-G, x)+(1-\lambda) p(-H, x) \geq p(-(\lambda G+(1-\lambda) H), x) .
$$

First if either $p(-G, x)=+\infty$ or $p(-H, x)=+\infty$, (??) is immediate (recall that $p(-G, x)>-\infty$ and $p(-H, x)>-\infty)$. So assume that $p(-G, x)$ and $p(-H, x)$ are both finite and fix some $\varepsilon>0$. Then setting $z_{G}=x+\varepsilon+p(-G, x)$ and $z_{H}=x+\varepsilon+p(-H, x)$, we get that

$$
\begin{aligned}
& u(0, x) \leq u\left(-G, z_{G}\right) \text { and } u(0, x) \leq u\left(-H, z_{H}\right) \\
& u(0, x) \leq \lambda u\left(-G, z_{G}\right)+(1-\lambda) u\left(-H, z_{H}\right),
\end{aligned}
$$

since both right-hand side are finite. It remains to prove that

$$
\lambda u\left(-G, z_{G}\right)+(1-\lambda) u\left(-H, z_{H}\right) \leq u\left(-(\lambda G+(1-\lambda) H), \lambda z_{G}+(1-\lambda) z_{H}\right) .
$$

Indeed (??) and (??) imply that $\varepsilon+\lambda p(-G, x)+(1-\lambda) p(-H, x) \geq p(-(\lambda G+(1-\lambda) H), x)$ and as this is true for all $\varepsilon>0$, the convexity of $\rho_{x}$ is proven.

The third part of the proposition follows from Proposition ?? under the additional assumption.

If $u\left(-G, z_{G}\right)=-\infty$ or $u\left(-H, z_{H}\right)=-\infty$ there is nothing to prove in (??) (recall that $u\left(-G, z_{G}\right)<+\infty$ and $\left.u\left(-H, z_{H}\right)<+\infty\right)$. So we assume that $u\left(-G, z_{G}\right)$ and $u\left(-H, z_{H}\right)$ are both finite. Recalling (??), there exists some $\phi_{G} \in \mathcal{A}\left(U,-G, z_{G}\right), \phi_{H} \in$ $\mathcal{A}\left(U,-H, z_{H}\right)$ such that

$$
\begin{gathered}
u\left(-G, z_{G}\right)-\varepsilon \leq \inf _{P \in \mathcal{Q}^{T}} E_{P} U\left(\cdot, V_{T}^{z_{G}, \phi_{G}}(\cdot)+G(\cdot)\right), \\
u\left(-H, z_{H}\right)-\varepsilon \leq \inf _{P \in \mathcal{Q}^{T}} E_{P} U\left(\cdot, V_{T}^{z_{H}, \phi_{H}}(\cdot)+H(\cdot)\right) .
\end{gathered}
$$

It follows that

$$
\begin{aligned}
\lambda u\left(-G, z_{G}\right)+(1-\lambda) u\left(-H, z_{H}\right)-\varepsilon & \leq \inf _{P \in \mathcal{Q}^{T}} E_{P} U\left(\cdot, V_{T}^{\lambda z_{G}+(1-\lambda) z_{H}, \lambda \phi_{G}+(1-\lambda) \phi_{H}}(\cdot)+\lambda G(\cdot)+(1-\lambda) H(\cdot)\right) \\
& \leq u\left(-(\lambda G+(1-\lambda) H), \lambda z_{G}+(1-\lambda) z_{H}\right),
\end{aligned}
$$

where we have used the concavity of $U$ and the fact that if $\phi_{G} \in \mathcal{A}\left(U,-G, z_{G}\right)=$ $\mathcal{A}\left(-G, z_{G}\right)$ and $\phi_{H} \in \mathcal{A}\left(U,-H, z_{H}\right)=\mathcal{A}\left(-H, z_{H}\right)$, then $\lambda \phi_{G}+(1-\lambda) \phi_{H} \in \mathcal{A}(-(\lambda G+$ $\left.(1-\lambda) H), \lambda z_{H}+(1-\lambda) z_{G}\right)=\mathcal{A}\left(U,-(\lambda G+(1-\lambda) H), \lambda z_{G}+(1-\lambda) z_{H}\right)$ by assumption. 
As the previous inequality is true for all $\varepsilon$, (??) is proven.

Proof of Proposition ??. 1. We set for all $y \geq 0, P \in \mathcal{Q}^{T} \psi_{P}(y)=E_{P} U(\cdot, y)$ and $\psi(y)=\inf _{P \in \mathcal{Q}^{T}} \psi_{P}(y)$. In the rest of the proof, the properties concerning $\psi_{P}$ will be stated for all $P \in \mathcal{Q}^{T}$. It is clear that $\psi_{P}$ is concave, strictly increasing and that $\psi$ is concave and non-decreasing. As for all $y>0, \sup _{P \in \mathcal{Q}^{T}} E_{P} U^{-}(\cdot, y)<+\infty$, we have that $\operatorname{Ri}(\operatorname{Dom} \psi)=\operatorname{Ri}\left(\operatorname{Dom} \psi_{P}\right)=(0,+\infty)$ (where $\operatorname{Dom} \psi=\{y, \psi(y)>-\infty\}$ and $\operatorname{Ri}(\operatorname{Dom} \psi)$ is its relative interior). So $\psi$ and $\psi_{P}$ are continuous on $(0, \infty)$. Using the monotonicity of $U$, for all $0 \leq y \leq 1 U(\cdot, y) \leq U^{+}(\cdot, 1)$ and as $E_{P} U^{+}(\cdot, 1)<+\infty$, the monotone convergence theorem applies and we get that $\lim _{y \searrow_{0}} \psi_{P}(y)=\psi_{P}(0)$. Thus the function $\psi_{P}$ is right-continuous in 0 and it follows easily that $\psi$ is also rightcontinuous in 0 .

Now let $F(\cdot):=\lim _{y \rightarrow+\infty} U(\cdot, y) \in(-\infty, \infty]$. Since $E_{P} U^{-}(\cdot, 1)<+\infty$ by assumption, the monotone convergence theorem applied and we get that

$$
\lim _{y \nearrow+\infty} \psi_{P}(y)=E_{P} F(\cdot) \in(-\infty,+\infty]
$$

As $P(G(\cdot)<\infty)=1$ and $U$ is strictly increasing

$$
F(\cdot)-U(\cdot, G(\cdot))>0 \text {-a.s. }
$$

1.a. Set for all $y \geq 0, \bar{\psi}_{P}(y)=\psi_{P}(y)-E_{P} U(\cdot, G(\cdot))$ which is well-defined since $E_{P}|U(\cdot, G(\cdot))|<+\infty$ for all $P \in \mathcal{Q}^{T}$. It is clear that $\bar{\psi}_{P}$ is continuous on $(0,+\infty)$ and right-continuous in 0 . Thus

$$
\bar{\psi}_{P}(0)=E_{P} U(\cdot, 0)-E_{P} U(\cdot, G(\cdot)) \leq 0,
$$

since $U$ is non-decreasing and $G \in \mathcal{W}_{T}^{0,+}$. As $E_{P} U(\cdot, G(\cdot)) \leq E_{P}|U(\cdot, G(\cdot))|<\infty$ by assumption, if $\lim _{y} \nearrow_{+\infty} \psi_{P}(y)=+\infty$ then for $y$ large enough $\bar{\psi}_{P}(y)>0$. Now if $\lim _{y \nearrow+\infty} \psi_{P}(y)<+\infty$, then (??) and (??) imply that $E_{P} U(\cdot, G(\cdot))<E_{P} F(\cdot)=$ $\lim _{y \nearrow+\infty} \psi_{P}(y)$ and $\bar{\psi}_{P}(y)>0$ again for some $y$ large enough. In both cases, the intermediate value theorem gives a unique $e(G, P) \in[0,+\infty)$ such that $\bar{\psi}_{P}(e(G, P))=0$ and (??) is proved.

1.b. Set $\bar{\psi}(y)=\psi(y)-\inf _{P \in \mathcal{Q}^{T}} E_{P} U(\cdot, G(\cdot))$ which is well-defined as $\sup _{P \in \mathcal{Q}^{T}} E_{P} U^{-}(\cdot, G(\cdot))<$ $\infty$ and $\inf _{P \in \mathcal{Q}^{T}} E_{P} U^{+}(\cdot, G(\cdot))<\infty$. The function $\bar{\psi}$ is continuous on $(0,+\infty)$ and rightcontinuous in 0 and using (??), we get that

$$
\inf _{P \in \mathcal{Q}^{T}} E_{P} U(\cdot, G(\cdot)) \geq \inf _{P \in \mathcal{Q}^{T}} E_{P} U(\cdot, 0)=\psi(0),
$$

$\bar{\psi}(0) \leq 0$ follows. Since $\inf _{P \in \mathcal{Q}^{T}} E_{P} U^{\prime}(\cdot, z)>0$ for all $z>0, \psi$ is strictly increasing on $(0,+\infty)$. Indeed let $0<x<y$, then $U(\cdot, x)+(y-x) U^{\prime}(\cdot, y) \leq U(\cdot, y)$ and this implies that

$$
\inf _{P \in \mathcal{Q}^{T}} E_{P} U(\cdot, x)+(y-x) \inf _{P \in \mathcal{Q}^{T}} E_{P} U^{\prime}(\cdot, y) \leq \inf _{P \in \mathcal{Q}^{T}} E_{P} U(\cdot, y) .
$$

As $G \in \mathcal{W}_{T}^{\infty,+}$, using the monotonicity of $U$ we obtain for any $\varepsilon>0$

$$
\inf _{P \in \mathcal{Q}^{T}} E_{P} U(\cdot, G(\cdot)) \leq \inf _{P \in \mathcal{Q}^{T}} E_{P} U\left(\cdot,\|G\|_{\infty}\right)=\psi\left(\|G\|_{\infty}\right)<\psi\left(\|G\|_{\infty}+\varepsilon\right)
$$


and thus $\bar{\psi}\left(\|G\|_{\infty}+\varepsilon\right)>0$. We apply again the intermediate value theorem and there exist a unique $e(G) \in\left[0,\|G\|_{\infty}\right]$ such that $\bar{\psi}(e(G))=0$ and (??) is proved. Now for any $Q \in \mathcal{Q}^{T}$, (??) implies that

$$
E_{Q} U\left(\cdot \inf _{P \in \mathcal{Q}^{T}} e(G, P)\right) \leq E_{Q} U(\cdot, e(G, Q))=E_{Q} U(\cdot, G(\cdot)) .
$$

Therefore using (??), $\psi\left(\inf _{P \in \mathcal{Q}^{T}} e(G, P)\right) \leq \psi(e(G))$ and $e(G) \geq \inf _{P \in \mathcal{Q}^{T}} e(G, P)$ follows since $\psi$ is strictly increasing.

2. From Definition ?? and $\operatorname{Dom}(U)=(0, \infty), U$ is continuous on $(0, \infty)$ and rightcontinuous in 0 . Fix some $P \in \mathcal{Q}^{T}$. As $E_{P} U^{-}(G(\cdot))<+\infty, G \in \mathcal{W}_{T}^{0,+}$ and $U$ is nondecreasing, $E_{P} U(G(\cdot))-U(0) \geq 0$ (recall that $U$ is non random). Now as before $P$-a.s $U\left(G\left(\omega^{T}\right)\right)<\lim _{y \rightarrow+\infty} U(y)$ and since $E_{P} U^{+}(G(\cdot))<+\infty$, one can always conclude that $E_{P} U(G(\cdot))-U(y)<0$ for $y$ large enough and the intermediate value theorem implies that (??) holds true.

Now since $E_{P} U(G(\cdot)) \geq U(0), \inf _{P \in \mathcal{Q}^{T}} E_{P} U(G(\cdot))-U(0) \geq 0$. Moreover as $\inf _{P \in \mathcal{Q}^{T}} E_{P} U(G(\cdot))-$ $U(y) \leq E_{P} U(G(\cdot))-U(y)<0$ for $y$ large enough, the intermediate value theorem implies (??). As before (see (??)) one can prove that $\inf _{P \in \mathcal{Q}^{T}} e(G, P) \leq e(G)$. For some $P \in \mathcal{Q}^{T}$ fixed, using Jensen's inequality we have that

$$
U(e(G)) \leq E_{P} U(G(\cdot))=U(e(G, P)) \leq U\left(E_{P} G(\cdot)\right) .
$$

Thus, by strict monotonicity of $U, e(G) \leq e(G, P) \leq E_{P} G(\cdot)$ and since this is true for all $P \in \mathcal{Q}^{T}$, we find that

$$
e(G) \leq \inf _{P \in \mathcal{Q}^{T}} e(G, P) \leq \inf _{P \in \mathcal{Q}^{T}} E_{P} G(\cdot)
$$

Note that it is easy to find an example where $\bar{\psi}$ is constant and thus $e(G)$ is not unique if $\inf _{P \in \mathcal{Q}^{T}} E_{P} U^{\prime}(\cdot, z)=0$

Proof of Proposition ??. We adapt the proof of (?, Proposition 2.47) to the multiplepriors framework.

1. We first show that if for all $x>0, r_{A}(x) \geq r_{B}(x)$, then $e_{A}(G, P) \leq e_{B}(G, P)$ for all $P \in \mathcal{Q}^{T}$ and $G \in \mathcal{W}_{T}^{+}(U)$. This will imply that $e_{A}(G) \leq e_{B}(G)$ using the second item of Proposition ??. We fix some $G \in \mathcal{W}_{T}^{+}(U)$ and $P \in \mathcal{Q}^{T}$. Let $D:=U_{B}((0, \infty)) \subset(-\infty, \infty)$ and define $F: D \rightarrow \mathbb{R}$ by $F(y)=U_{A}\left(U_{B}^{-1}(y)\right)$. Then on $D$

$$
F^{\prime}(\cdot)=\frac{U_{A}^{\prime}\left(U_{B}^{-1}(\cdot)\right)}{U_{B}^{\prime}\left(U_{B}^{-1}(\cdot)\right)} \text { and } F^{\prime \prime}(\cdot)=\frac{U_{A}^{\prime}\left(U_{B}^{-1}(\cdot)\right)}{\left(U_{B}^{\prime}\left(U_{B}^{-1}(\cdot)\right)\right)^{2}}\left(r_{B}\left(U_{B}^{-1}(\cdot)\right)-r_{A}\left(U_{B}^{-1}(\cdot)\right)\right) \text {. }
$$

As $U_{B}^{-1}(\cdot)>0$ on $D, F$ is increasing and concave on $D$ and $U_{A}(x)=F\left(U_{B}(x)\right)$ for all $x>0$. Now let $d:=U_{B}(0) \in[-\infty, \infty)$ be the lower bound of $D$. We distinguish between two cases. First if $d>-\infty$, we extend $F$ by continuity in $d$, setting $F(d)=$ $U_{A}\left(U_{B}^{-1}(d)\right)=U_{A}(0) \in[-\infty, \infty)$. It is clear that $F(d) \leq F(y)$ for all $y \in[d,+\infty)$, that $F$ is concave on $[d,+\infty)$ and that $U_{A}(x)=F\left(U_{B}(x)\right)$ holds also true for all $x \geq 0$. Now, using the fact that $U_{A}$ and $U_{B}$ are non-random, (??) and Jensen's inequality, we get that

$U_{A}\left(e_{A}(G, P)\right)=E_{P} U_{A}(G(\cdot))=E_{P} F\left(U_{B}(G(\cdot))\right) \leq F\left(E_{P}\left(U_{B}(G(\cdot))\right)\right)=F\left(U_{B}\left(e_{B}(G, P)\right)\right)=U_{A}\left(e_{B}(G, P)\right)$. 
Since $U_{A}$ is strictly increasing, we obtain that $e_{A}(G, P) \leq e_{B}(G, P)$ as claimed.

Now we treat the case where $d=-\infty$. First $P(G>0)=1$. Indeed if $P(G=0)>0$, $E_{P} U_{B}^{-}(G(\cdot))=E_{P} U_{B}^{-}(G(\cdot)) 1_{\{G>0\}}(\cdot)+U_{B}^{-}(0) P(G=0)=+\infty$, a case that we have excluded. Thus $P(G>0)=1$, the previous arguments apply and we also obtain $e_{A}(G, P) \leq e_{B}(G, P)$.

2. Assume that $e_{A}(G)<e_{B}(G)$ for all $G \in \mathcal{W}_{T}^{+}(U)$ and there exists some $x_{0}>0$ such that $r_{A}\left(x_{0}\right)<r_{B}\left(x_{0}\right)$. By continuity, there exists $\alpha>0$, such that $r_{A}(x)<r_{B}(x)$ on $\left(x_{0}-\alpha, x_{0}+\alpha\right)$. We can choose $\alpha$ such that $x_{0}-\alpha>0$. Let $I:=\left(U_{B}\left(x_{0}-\alpha\right), U_{B}\left(x_{0}+\alpha\right)\right) \subset$ $D$, then $F$ is strictly convex on $I$ (see (??)). Fix $\widetilde{G} \in \mathcal{W}_{T}^{+}(U)$ and set $G:=x_{0}-\alpha+$ $2 \alpha \frac{\widetilde{G}}{\widetilde{G}+1} \in \mathcal{W}_{T}^{+}(U)$. It is clear that $G(\cdot) \in\left(x_{0}-\alpha, x_{0}+\alpha\right)$. As in (??), using Jensen inequality, the fact that $F$ is (strictly) convex on $I$ we get that for any $P \in \mathcal{Q}^{T}$

$$
U_{A}\left(e_{A}(G, P)\right)=E_{P} F\left(U_{B}(G(\cdot))\right) \geq F\left(E_{P}\left(U_{B}(G(\cdot))\right)=F\left(U_{B}\left(e_{B}(G, P)\right)\right)=U_{A}\left(e_{B}(G, P)\right) .\right.
$$

This implies that $e_{A}(G, P) \geq e_{B}(G, P)$ for all $P \in \mathcal{Q}^{T}$, thus $e_{A}(G) \geq e_{B}(G)$ : a contradiction. Note that if $P$ is such that one can find some $\widetilde{G}$ which is not constant then the first inequality in (??) is strict and one gets that $e_{A}(G, P)>e_{B}(G, P)$.

Proof of Proposition ??. We use similar arguments as in the proof of (?, Theorem 4.17) and (?, Lemma 5.7). We fix $x \geq 0, \phi=\left(\phi_{t}\right)_{1<t<T} \in \Phi$ such that $\phi \in \mathcal{A}(0, x)$. For all $1 \leq t \leq T$ and $\omega^{t-1} \in \Omega_{N A}^{t-1}$, we denote by $\phi_{t}^{\perp}\left(\omega^{t-1}\right)$ the orthogonal projection of $\phi_{t}\left(\omega^{t-1}\right)$ on the vector space $D^{t}\left(\omega^{t-1}\right)$ (recall Proposition ??). We have for all $\omega^{t-1} \in \Omega_{N A}^{t-1}$, that

$$
\phi_{t}\left(\omega^{t-1}\right) \Delta S_{t}\left(\omega^{t-1}, \cdot\right)=\phi_{t}^{\perp}\left(\omega^{t-1}\right) \Delta S_{t}\left(\omega^{t-1}, \cdot\right) \mathcal{Q}_{t}\left(\omega^{t-1}\right) \text {-q.s. }
$$

see (?, Remark 3.10). As $V_{T}^{x, \phi} \geq 0 \mathcal{Q}^{T}$-q.s. and as Assumptions ??, ?? and ?? hold true, (?, Lemma 4.3) applies together with (?, Lemma 3.4) and we obtain that the set $\mathcal{H}^{t-1}:=\left\{\omega^{t-1} \in \Omega^{t-1}, V_{t-1}^{x, \phi}\left(\omega^{t-1}\right)+\phi_{t}\left(\omega^{t-1}\right) \Delta S_{t}\left(\omega^{t-1}, \cdot\right) \geq 0 \mathcal{Q}_{t}\left(\omega^{t-1}\right)\right.$-q.s. $\}$ is a $\mathcal{Q}^{t-1}$-full measure set. We fix now some $1 \leq t \leq T$, $\omega^{t-1} \in \mathcal{H}^{t-1} \cap \Omega_{N A}^{t-1}$ and we prove that

$$
\left|\phi_{t}^{\perp}\left(\omega^{t-1}\right)\right| \leq \frac{\left|V_{t-1}^{x, \phi}\left(\omega^{t-1}\right)\right|}{\alpha_{t-1}\left(\omega^{t-1}\right)} .
$$

If $\phi_{t}^{\perp}\left(\omega^{t-1}\right)=0$ there is nothing to prove. So we can assume that $\phi_{t}^{\perp}\left(\omega^{t-1}\right) \neq 0$. First, using (??), since $\omega^{t-1} \in \mathcal{H}^{t-1} \cap \Omega_{N A}^{t-1}$, we get that

$$
V_{t-1}^{x, \phi}\left(\omega^{t-1}\right)+\phi_{t}^{\perp}\left(\omega^{t-1}\right) \Delta S_{t}\left(\omega^{t-1}, \cdot\right) \geq 0 \mathcal{Q}_{t}\left(\omega^{t-1}\right) \text {-q.s. }
$$

Now, we proceed by contradiction and assume that (??) does not hold true. We set $B:=\left\{\phi_{t}^{\perp}\left(\omega^{t-1}\right) \Delta S_{t}\left(\omega^{t-1}, \cdot\right)<-\alpha_{t-1}\left(\omega^{t-1}\right)\left|\phi_{t}^{\perp}\left(\omega^{t-1}\right)\right|\right\}$. From Proposition ??, there exists some $P_{\phi} \in \mathcal{Q}_{t}\left(\omega^{t-1}\right)$ such that $P_{\phi}(B)>\alpha_{t-1}\left(\omega^{t-1}\right)>0$. But, for all $\omega_{t} \in B$ we have that

$$
V_{t-1}^{x, \phi}\left(\omega^{t-1}\right)+\phi_{t}^{\perp}\left(\omega^{t-1}\right) \Delta S_{t}\left(\omega^{t-1}, \omega_{t}\right)<\left|V_{t-1}^{x, \phi}\left(\omega^{t-1}\right)\right|-\alpha_{t-1}\left(\omega^{t-1}\right)\left|\phi_{t}^{\perp}\left(\omega^{t-1}\right)\right|<0,
$$

a contradiction with (??) and therefore (??) holds true.

We now establish (??) by induction. For $t=0$ this is trivial. Assume now that for some $t \geq 1$, there exists some $\mathcal{Q}^{t-1}$-full measure set $\widetilde{\Omega}^{t-1} \in \mathcal{B}_{c}\left(\Omega^{t-1}\right)$ on which (??) is true at stage $t-1$. We denote by

$$
\Omega_{E Q}^{t}:=\left\{\left(\omega^{t-1}, \omega_{t}\right) \in \Omega^{t-1} \times \Omega_{t}, \phi_{t}^{\perp}\left(\omega^{t-1}\right) \Delta S_{t}\left(\omega^{t-1}, \omega_{t}\right)=\phi_{t}\left(\omega^{t-1}\right) \Delta S_{t}\left(\omega^{t-1}, \omega_{t}\right)\right\} .
$$


It is clear that $\Omega_{E Q}^{t} \in \mathcal{B}_{c}\left(\Omega^{t}\right)$. For some $P=P_{t-1} \otimes p_{t} \in \mathcal{Q}^{t}$, recalling (??) and applying Fubini's Theorem (see (?, Proposition 7.45 p175)), we have that

$$
\begin{aligned}
P\left(\Omega_{E Q}^{t}\right) & =\int_{\Omega^{t-1}} \int_{\Omega_{t}} 1_{\Omega_{E Q}^{t}}\left(\omega^{t-1}, \omega_{t}\right) p_{t}\left(d \omega_{t}, \omega^{t-1}\right) P_{t-1}\left(d \omega^{t-1}\right) \\
& =\int_{\Omega_{N A}^{t-1}} \int_{\Omega_{t}} p_{t}\left(\phi_{t}^{\perp}\left(\omega^{t-1}\right) \Delta S_{t}\left(\omega^{t-1}, \cdot\right)=\phi_{t}\left(\omega^{t-1}\right) \Delta S_{t}\left(\omega^{t-1}, \cdot\right), \omega^{t-1}\right) P_{t-1}\left(d \omega^{t-1}\right) \\
& =1 .
\end{aligned}
$$

Set $\widehat{\Omega}^{t-1}:=\widetilde{\Omega}^{t-1} \cap \mathcal{H}^{t-1} \cap \Omega_{N A}^{t-1}$ and $\widetilde{\Omega}^{t}=\Omega_{E Q}^{t} \cap\left(\widehat{\Omega}^{t-1} \times \Omega_{t}\right)$. It is clear that $\widetilde{\Omega}^{t} \in \mathcal{B}_{c}\left(\Omega^{t}\right)$ and is a $\mathcal{Q}^{t}$-full measure set. We have that for all $\omega^{t}=\left(\omega^{t-1}, \omega_{t}\right) \in \widetilde{\Omega}^{t}$

$$
\begin{aligned}
\left|V_{t}^{x, \phi}\left(\omega^{t-1}, \omega_{t}\right)\right| & =\left|V_{t-1}^{x, \phi}\left(\omega^{t-1}\right)+\phi_{t}^{\perp}\left(\omega^{t-1}\right) \Delta S_{t}\left(\omega^{t-1}, \omega_{t}\right)\right| \\
& \leq\left|V_{t-1}^{x, \phi}\left(\omega^{t-1}\right)\right|\left(1+\frac{\left|\Delta S_{t}\left(\omega^{t-1}, \omega_{t}\right)\right|}{\alpha_{t-1}\left(\omega^{t-1}\right)}\right) \\
& \leq x M_{t-1}\left(\omega^{t-1}\right)\left(1+\frac{\left|\Delta S_{t}\left(\omega^{t-1}, \omega_{t}\right)\right|}{\alpha_{t-1}\left(\omega^{t-1}\right)}\right)
\end{aligned}
$$

where we have used the fact that $\omega^{t} \in \Omega_{E Q}^{t}$ for the first equality, $\omega^{t-1} \in \mathcal{H}^{t-1} \cap \Omega_{N A}^{t-1}$ and (??) for the second inequality and $\omega^{t-1} \in \widetilde{\Omega}^{t-1}$ for the last one: (??) is proved.

For all $0 \leq r<\infty$ and $1 \leq s \leq T$, as $\Delta S_{s}, \frac{1}{\alpha_{s}} \in \mathcal{W}_{s}^{r}$ (see Assumption ??), so both $M_{t}$ and $V_{t}^{x, \phi}$ belong to $\mathcal{W}_{t}^{r}$ for all $1 \leq t \leq T$.

Fix now some $n \geq 1$. Using the monotonicity, concavity and differentiability of $U_{n}\left(\omega^{T}, \cdot\right)$, we get for all $\omega^{T} \in \Omega^{T}$ that

$$
U_{n}\left(\omega^{T}, x\right) \leq U_{n}\left(\omega^{T}, \max \left(x, x_{0}\right)\right) \leq U_{n}\left(\omega^{T}, x_{0}\right)+\max \left(x-x_{0}, 0\right) U_{n}^{\prime}\left(\omega^{T}, x_{0}\right) .
$$

Thus

$$
U_{n}^{+}\left(\omega^{T}, x\right) \leq U_{n}^{+}\left(\omega^{T}, x_{0}\right)+|x| U_{n}^{\prime}\left(\omega^{T}, x_{0}\right) .
$$

And it follows that for all $P \in \mathcal{Q}^{T}$

$$
\begin{aligned}
E_{P} U_{n}^{+}\left(\omega^{T}, V_{T}^{x, \phi}\left(\omega^{T}\right)\right) & \leq \sup _{P \in \mathcal{Q}^{T}} E_{P} U_{n}^{+}\left(\cdot, x_{0}\right)+\sup _{P \in \mathcal{Q}^{T}} E_{P}\left(\left|V_{T}^{x, \phi}(\cdot)\right| U_{n}^{\prime}\left(\cdot, x_{0}\right)\right) \\
& \leq \sup _{P \in \mathcal{Q}^{T}} E_{P} U_{n}^{+}\left(\cdot, x_{0}\right)+x\left(\sup _{P \in \mathcal{Q}^{T}} E_{P}\left(M_{T}(\cdot)\right)^{p}\right)^{\frac{1}{p}}\left(\sup _{P \in \mathcal{Q}^{T}} E_{P}\left(U_{n}^{\prime}\left(\cdot, x_{0}\right)\right)^{q}\right)^{\frac{1}{q}} \\
& \leq \sup _{n}\left\|U_{n}^{+}\left(\cdot, x_{0}\right)\right\|_{1}+x\left\|M_{T}(\cdot)\right\|_{p} \sup _{n}\left\|U_{n}^{\prime}\left(\cdot, x_{0}\right)\right\|_{q}=K_{x}<\infty,
\end{aligned}
$$

where we have used (??), (??), $M_{T} \in \mathcal{W}_{T}^{r}$ for all $r \geq 1$, Assumption ??, (?, Proposition 16) ( $p$ verifies $\frac{1}{p}+\frac{1}{q}=1$ ) and finally, again Assumption ?? for the last inequality. As this is true for all $P \in \mathcal{Q}^{T}$ and as $K_{x}$ does not depend on $P$ and $n$, (??) is proved.

Proposition 5.1 Let $\left(X_{n}\right)_{n \geq 1}$ and $X$ be $\mathbb{R}^{d}$-valued and $\mathcal{B}_{c}\left(\Omega^{T}\right)$-measurable random variables. If $\lim _{n \rightarrow \infty} \sup _{P \in \mathcal{Q}^{T}} P\left(\left|X_{n}-X\right|>\frac{1}{n}\right)=0$, then there exists a subsequence $\left(X_{n_{k}}\right)_{k \geq 1}$ that converges to $X \mathcal{Q}^{T}$-q.s. (i.e. on a $\mathcal{Q}^{T}$-full measure set). 
Proof. Fix $\varepsilon>0$ and consider the sub-sequence $\left(X_{n_{k}}\right)_{k \geq 1}$ such that $\sup _{P \in \mathcal{Q}^{T}} P\left(A_{k}\right) \leq$ $\frac{1}{2^{k}}$ where $A_{k}:=\left\{\left|X_{n_{k}}(\cdot)-X(\cdot)\right|>\frac{1}{\max \left(n_{k}, \varepsilon\right)}\right\}$. As $\sum_{k \geq 1} \sup _{P \in \mathcal{Q}^{T}} P\left(A_{k}\right)<\infty$, using Borel-Cantelli's Lemma for capacity (see (?, Lemma 5)), we get that $\sup _{P \in \mathcal{Q}^{T}} P\left(\limsup _{k} A_{k}\right)=$ 0 . Hence $\Omega^{T} \backslash \lim \sup _{k} A_{k}$ is a $\mathcal{Q}^{T}$-full measure set on which $\left|X_{n_{k}}(\cdot)-X(\cdot)\right| \leq \varepsilon$ holds true for $k$ big enough. 